\title{
Semitopologization in motivic homotopy theory and applications
}

\author{
AMALENDU KRISHNA \\ JINHYUN PARK
}

\begin{abstract}
We study the semitopologization functor of Friedlander and Walker from the perspective of motivic homotopy theory. We construct a triangulated endofunctor on the stable motivic homotopy category $\mathcal{S H}(\mathbb{C})$, which we call homotopy semitopologization. As applications, we discuss the representability of several semitopological cohomology theories in $\mathcal{S H}(\mathbb{C})$, a construction of a semitopological analogue of algebraic cobordism and a construction of Atiyah-Hirzebruch type spectral sequences for this theory.
\end{abstract}

14F42; 19E08

\section{Introduction}

The goal of this paper is to study semitopological cohomology theories such as semitopological $K$-theory of Friedlander and Walker [9] and morphic cohomology of Friedlander and Lawson [5] from the perspective of motivic homotopy theory. One feature of the semitopological theories is that they can be obtained as semitopologizations of other theories, such as motivic cohomology or algebraic $K$-theory, as pioneered by Friedlander and Walker [8], but semitopologization does not respect all motivic weak-equivalences, so that it is not an endofunctor on motivic homotopy categories. Nonetheless, we show that it induces a derived functor, call it homotopy semitopologization, using the fact that semitopologization does respect at least objectwise weak-equivalences (see Section 5.1). So we first ask when a motivic weak-equivalence may become an objectwise one. After a review of motivic homotopy theory in Section 2, we answer that question in Section 3:

Theorem 1.0.1 A motivic weak-equivalence $E \rightarrow F$ of $\mathbb{A}^{1}-B G$ presheaves on $\mathbf{S m}_{S}$ is an objectwise weak-equivalence. Let $T=\left(\mathbb{P}^{1}, \infty\right)$. A stable motivic weak-equivalence $E \rightarrow F$ of $\mathbb{A}^{1}-B G$ motivic $\Omega_{T}$-bispectra on $\mathbf{S m}_{S}$ is a $T$-levelwise objectwise weakequivalence. 
In Sections 4 and 5, we show that these objects where motivic weak-equivalences behave well are closed under the semitopologization, and we define in Section 6 the derived functor on the stable motivic homotopy category $\mathcal{S H}(\mathbb{C})$ :

Theorem 1.0.2 There is a triangulated endofunctor host: $\mathcal{S H}(\mathbb{C}) \rightarrow \mathcal{S H}(\mathbb{C})$ that coincides with Friedlander-Walker semitopologization on $\mathbb{A}^{1}-B G$ motivic $\Omega_{T}$-bispectra.

Using host, in Sections 7 and 8 we prove the representability of the semitopological $K$-theory and the morphic cohomology in $\mathcal{S H}(\mathbb{C})$. In Section 9 we define a semitopological analogue of the algebraic cobordism of Voevodsky [41] by simply homotopy semitopologizing MGL:

Theorem 1.0.3 The semitopological $K$-theory and the morphic cohomology are representable in $\mathcal{S H}(\mathbb{C})$. There is a semitopological cobordism $\mathrm{MGL}_{\text {sst }}$ as a cohomology theory on $\mathbf{S m}_{\mathbb{C}}$, with a natural transformation MGL $\rightarrow \mathrm{MGL}_{\text {sst }}$ that becomes an isomorphism with finite coefficients. For $X \in \mathbf{S m}_{\mathbb{C}}$ and $n \geq 0$, there is a spectral sequence $E_{2}^{p, q}(n)=L^{n-q} H^{p-q}(X) \otimes_{\mathbb{Z}} \mathbb{L}^{q} \Rightarrow \operatorname{MGL}_{\text {sst }}^{p+q, n}(X)$. This spectral sequence degenerates after tensoring with $\mathbb{Q}$.

Conventions and notation When $S$ is a noetherian scheme of finite Krull dimension, an $S$-scheme is a separated scheme of finite type over $S$. The category of $S$-schemes is $\mathbf{S c h}_{S}$, while its subcategory of smooth schemes is $\mathbf{S m}_{S}$. A variety over $k$ is a reduced $k$-scheme, not necessarily quasiprojective. The category of $k$-varieties is $\operatorname{Var}_{k}$.

Let Set, Spc and Spc. be the categories of sets, simplicial sets and pointed simplicial sets. Let Spt be the category of Bousfield-Friedlander spectra [2] (see Section 2.2). The set of maps $K \rightarrow L$ in $\mathbf{S p c}_{\bullet}$ is $\operatorname{Hom}_{\bullet}(K, L)$.

The symbol $\Delta$ is used for the following, and no confusion should arise. First, $\Delta$ is the category whose objects are $[n]:=\{0, \ldots, n\}$ for $n \geq 0$ and the morphisms are nondecreasing set functions. The notation $\Delta[n]$ is the simplicial set $\operatorname{Hom}_{\text {Set }}(-,[n])$ by Yoneda. The notation $\Delta_{\top}^{n}$ is the topological $n$-simplex $\left\{\left(t_{0}, \ldots, t_{n}\right) \in \mathbb{R}^{n+1} \mid 0 \leq t_{i} \leq\right.$ $\left.1, \sum_{i} t_{i}=1\right\}$, while $\Delta^{n}$ is the algebraic $n$-simplex $\operatorname{Spec}\left(k\left[t_{0}, \ldots, t_{n}\right] / \sum_{i} t_{i}-1\right)$.

\section{Recollection of motivic homotopy theory}

We review basics of motivic homotopy theory from Jardine [18], Morel and Voevodsky [32] and Morel [30]. Throughout Sections 2 and 3, let $S$ be a fixed noetherian scheme of finite Krull dimension. 


\subsection{Motivic spaces}

We regard an object of Spc as a space, that of Spc. as a pointed space. A motivic space over $S$ is a simplicial presheaf on $\mathbf{S m}_{S}$. A pointed motivic space is a pointed simplicial presheaf on $\operatorname{Sm}_{S}$. Let $\operatorname{Spc}(S)$ and $\operatorname{Spc}_{\bullet}(S)$ be the categories of unpointed and pointed motivic spaces. A presheaf of sets on $\mathbf{S m}_{S}$ is a motivic space of simplicial dimension zero. Each $X \in \mathbf{S m}_{S}$ is a motivic space by Yoneda embedding. By $X_{+}$, we mean $X \amalg S \in \mathbf{S p c}_{\bullet}(S)$. Each (pointed) space $K$ is a (pointed) motivic space, being a constant presheaf on $\mathbf{S m}_{S}$. For $U \in \mathbf{S p c}_{\bullet}(S)$, the suspension $\Sigma_{U}$ : $\mathbf{S p c}_{\bullet}(S) \rightarrow \mathbf{S p c}_{\bullet}(S)$ sends $E$ to $E \wedge U$. For $U=S^{1},\left(\mathbb{G}_{m},\{1\}\right)$ and $T=\left(\mathbb{P}^{1}, \infty\right)$, we write $\Sigma_{U}$ as $\Sigma_{s}, \Sigma_{t}$ and $\Sigma_{T}$. For $E, F$ in $\operatorname{Spc}(S)$ and in $\operatorname{Spc}_{\bullet}(S)$, let $\mathcal{H o m}(E, F)$ and $\mathcal{H o m}_{\bullet}(E, F)$ be the internal hom presheaves of objects in $\mathbf{S p c}$ and $\mathbf{S p c}_{\bullet}$. For $E \in \mathbf{S p c}_{\bullet}(S)$, the functor $\mathcal{H o m}_{\bullet}(E,-)$ on Spc $\mathbf{S c}_{\bullet}$ is denoted by $\Omega_{E}(-)$. For $E=S^{1}$ and $\left(\mathbb{G}_{m}, 1\right)$, we write $\Omega_{E}(-)$ as $\Omega_{s}(-)$ and $\Omega_{t}(-)$.

\section{2 $S^{1}$-stable motivic homotopy category}

Recall (see [18, Theorem 1.1]) that $\mathbf{S p c}(S)$ is a proper simplicial cellular closed model category, where a map $f: E \rightarrow F$ is a Nisnevich local weak-equivalence if all induced Nisnevich stalk maps $E_{x} \rightarrow F_{x}$ are weak-equivalences of Spc, while cofibrations are monomorphisms, and Nisnevich fibrations are defined in terms of the right lifting property with respect to all trivial cofibrations. A similar model structure on $\mathbf{S p c}_{\bullet}(S)$ exists. Inverting the Nisnevich local weak-equivalences, we get the homotopy categories $\mathcal{H}^{\mathrm{Nis}}(S)$ and $\mathcal{H}_{\bullet}^{\mathrm{Nis}}(S)$. For $E, F \in \mathbf{S p c}_{\bullet}(S)$, let $[E, F]_{\mathrm{Nis}}:=\operatorname{Hom}_{\mathcal{H}_{\bullet}^{\mathrm{Nis}}(S)}(E, F)$. See $[18 ; 32]$ for more details.

A spectrum, or an $S^{1}$-spectrum, is a sequence $\left(E_{0}, E_{1}, \ldots\right), E_{i} \in \mathbf{S p c}_{\bullet}$, with morphisms $S^{1} \wedge E_{n} \rightarrow E_{n+1}$ in $\mathbf{S p c} \bullet$. The category of spectra is $\mathbf{S p t}$, and the category of presheaves of spectra on $\operatorname{Sm}_{S}$ is $\operatorname{Spt}(S)$. An object of $\operatorname{Spt}(S)$ is called a motivic spectrum.

2.2.1 Nisnevich model structure on motivic spectra over $S$ A morphism $f: E \rightarrow$ $F$ in $\operatorname{Spt}(S)$ is an objectwise weak-equivalence if for each $U \in \mathbf{S m}_{S}$ the map $f(U): E(U) \rightarrow F(U)$ is an $S^{1}$-stable weak-equivalence in Spt. A morphism $f: E \rightarrow F$ in $\operatorname{Spt}(S)$ is a Nisnevich local weak-equivalence if for each $U \in \mathbf{S m}_{S}$ and $x \in U$ the induced map $f_{x}: E_{x} \rightarrow F_{x}$ on the Nisnevich stalks is an $S^{1}$-stable weak-equivalence in Spt. A map $f: E \rightarrow F$ in $\mathbf{S p t}(S)$ is a cofibration if $f_{0}$ is a monomorphism and $E_{n+1} U_{S^{1} \wedge E_{n}} S^{1} \wedge F_{n} \rightarrow F_{n+1}$ is a monomorphism in $\operatorname{Spc}(S)$ for each $n \geq 0$. Equivalently, the maps $E_{n} \rightarrow F_{n}$ and $S^{1} \wedge\left(F_{n} / E_{n}\right) \rightarrow F_{n+1} / E_{n+1}$ are monomorphisms in $\operatorname{Spc}(S)$ for each $n \geq 0$. A Nisnevich fibration in $\operatorname{Spt}(S)$ is a map 
with the right lifting property with respect to all trivial cofibrations. Giving a cofibration $E \rightarrow F$ in $\operatorname{Spt}(S)$ is equal to giving cofibrations $E(U) \rightarrow F(U)$ in Spt. A morphism $E \rightarrow F$ in $\operatorname{Spt}(S)$ is a Nisnevich local weak-equivalence if and only if the induced map of Nisnevich sheaves associated to the presheaves $U \mapsto \pi_{n}(E(U)), \pi_{n}(F(U))$, is an isomorphism for all $n \in \mathbb{Z}$. Recall:

Theorem 2.2.1 (Jardine [17, Theorem 2.34] and Morel [30, Lemma 2.3.6]) The above Nisnevich local weak-equivalences, cofibrations and Nisnevich fibrations define a proper simplicial closed model structure on $\operatorname{Spt}(S)$. An object $E$ is cofibrant if and only if the maps $S^{1} \wedge E_{n} \rightarrow E_{n+1}$ are monomorphisms. An object $E$ is Nisnevich fibrant if and only if each $E_{n}$ is a Nisnevich fibrant pointed motivic space and the adjoint maps $E_{n} \rightarrow \Omega_{s}^{1} E_{n+1}$ are Nisnevich local weak-equivalences.

For each $E \in \mathbf{S p c}_{\bullet}(S)$, the motivic spectrum $\Sigma_{s}^{\infty} E=\left(E, \Sigma_{s}^{1} E, \Sigma_{s}^{2} E, \ldots\right)$ is cofibrant. The homotopy category with respect to the above Nisnevich local injective model structure is $\mathcal{S H}_{S^{1}}^{\mathrm{Nis}}(S)$. For $E, F \in \operatorname{Spt}(S)$, let $[E, F]_{\mathrm{Nis}}:=\operatorname{Hom}_{\mathcal{S H}_{\mathcal{H}^{1}}^{\mathrm{Nis}}}(S)(E, F)$.

\subsubsection{Motivic model structure on motivic spectra over $S$ The homotopy category} with respect to the motivic model structure (see [18]) on $\mathbf{S p c}_{\bullet}(S)$ is denoted by $\mathcal{H}_{\bullet}(S)$, and we let $[E, F]_{\mathbb{A}^{1}}:=\operatorname{Hom}_{\mathcal{H} \bullet}(S)(E, F)$. We recall from [30, Section 4], the motivic model structure on $\operatorname{Spt}(S)$. We say $Z \in \operatorname{Spt}(S)$ is $\mathbb{A}^{1}$-local if for any $E \in \operatorname{Spt}(S)$, the projection $E \wedge \mathbb{A}_{+}^{1} \rightarrow E$ induces an isomorphism of groups $[E, Z]_{\mathrm{Nis}} \simeq\left[E \wedge \mathbb{A}_{+}^{1}, Z\right]_{\mathrm{Nis}}$. A morphism $f: E \rightarrow F$ in $\operatorname{Spt}(S)$ is an $S^{1}$-stable motivic weak-equivalence if for each $\mathbb{A}^{1}$-local $Z$, the induced map $f^{*}:[F, Z]_{\mathrm{Nis}} \rightarrow[E, Z]_{\mathrm{Nis}}$ is an isomorphism. We often say that $f$ is a motivic weak-equivalence of motivic spectra, for simplicity. The motivic weak-equivalences, cofibrations (as in Section 2.2.1) and motivic fibrations (given by the right lifting property with respect to all trivial cofibrations) define a closed model structure on $\operatorname{Spt}(S)$, called the $S^{1}$-stable motivic model structure. This model structure is the left localization of the Nisnevich local injective model structure with respect to the maps $E \wedge \mathbb{A}_{+}^{1} \rightarrow E$ for $E \in \operatorname{Spt}(S)$. By Hirschhorn [14, Proposition 3.4] and Theorem 2.2.1, the motivic model structure on $\operatorname{Spt}(S)$ is proper and simplicial. A motivic spectrum is motivic fibrant if and only if it is Nisnevich fibrant and $\mathbb{A}^{1}$-local. Let $\mathcal{S H}_{S^{1}}(S)$ be the homotopy category of $\operatorname{Spt}(S)$ with respect to the $S^{1}$-stable motivic model structure. This model structure is equivalent to the one obtained by stabilizing the motivic model structure on $\operatorname{Spc}_{\bullet}(S)$ with respect to $\Sigma_{S}$, as described in [18, Theorem 1.1]. It follows that $E \in \operatorname{Spt}(S)$ is motivic fibrant if and only if it is levelwise motivic fibrant in the motivic model structure on $\mathbf{S p c}_{\bullet}(S)$, and each map $E_{n} \rightarrow \Omega_{s} E_{n+1}$ is a motivic weak-equivalence. By [30, Proposition 3.1.1], the category $\mathcal{S H}_{S^{1}}(S)$ is triangulated, where the shift functor $E \mapsto E[1]$ is $\Sigma_{s}$. We let $[E, F]_{\mathbb{A}^{1}}:=$ 
$\operatorname{Hom}_{\mathcal{S H}_{S^{1}}(S)}(E, F)$. We have a pair of adjoint functors $\Sigma_{S}^{\infty}: \mathbf{S p c}_{\bullet}(S) \leftrightarrow \mathbf{S p t}(S): \operatorname{Ev}_{S}^{0}$ given by $\Sigma_{S}^{\infty}(E)=\left(E, \Sigma_{S} E, \Sigma_{S}^{2} E, \ldots\right)$ and $\operatorname{Ev}_{s}^{0}(F)=F_{0}$. The functor $\Sigma_{S}^{\infty}$ clearly preserves cofibrations. For $E \in \mathbf{S p c}_{\bullet}(S), F \in \mathbf{S p t}(S)$ and $p \in \mathbb{Z}$, there are natural isomorphisms (cf [41, Theorem 5.2])

$$
\left[\Sigma_{s}^{\infty} E[p], F\right]_{?} \simeq \operatorname{colim}_{n \geq-p}\left[S^{n+p} \wedge E, F_{n}\right]_{?}, \quad ?=\text { Nis or } \mathbb{A}^{1},
$$

so that the functor $\Sigma_{S}^{\infty}$ preserves motivic weak-equivalences. Thus, the pair $\left(\Sigma_{S}^{\infty}, \operatorname{Ev}_{s}^{0}\right)$ forms a Quillen pair, and one has adjoint functors $\Sigma_{s}^{\infty}: \mathcal{H}_{\bullet}(S) \leftrightarrow \mathcal{S H}_{S^{1}}(S): \boldsymbol{R}_{\mathrm{Ev}_{s}^{0}}^{0}$.

\subsection{Stable motivic homotopy category}

The stable motivic homotopy category $\mathcal{S H}(S)$ was first constructed in [41]. It has several models. We review two such models. For $F \in \operatorname{Spt}(S)$ and $E \in \operatorname{Spc}_{\bullet}(S)$, we let $\Sigma_{E} F$ denote the motivic spectrum $\left(F_{0} \wedge E, F_{1} \wedge E, \ldots\right)$. For $E=S^{1}$, the spectrum $\Sigma_{E} F$ is denoted by $\Sigma_{s} F$. Let $T=\left(\mathbb{P}^{1}, \infty\right)$.

2.3.1 $(s, \mathfrak{p})$-bispectra model Recall from Levine [23, Section 8] that an $(s, \mathfrak{p})-$ bispectrum over $S$ is a collection $E=\left\{E_{m, n} \in \mathbf{S p c}_{\bullet}(S) \mid m, n \geq 0\right\}$ with horizontal maps $\Sigma_{s} E_{m, n} \rightarrow E_{m+1, n}$ and vertical maps $\Sigma_{T} E_{m, n} \rightarrow E_{m, n+1}$ such that the horizontal and the vertical maps commute. We regard it as a sequence $\left(E_{0}, E_{1}, \ldots\right)$, with the bonding maps $\Sigma_{T} E_{n} \rightarrow E_{n+1}$, where $E_{n} \in \operatorname{Spt}(S)$ is $E_{*, n}:=\left(E_{0, n}, E_{1, n}, \ldots\right)$. Let $\operatorname{Spt}_{(s, \mathfrak{p})}(S)$ be the category of $(s, \mathfrak{p})$-bispectra over $S$. Given $E \in \mathbf{S p t}_{(s, \mathfrak{p})}(S)$ and $p, q \in \mathbb{Z}$, define $\pi_{p, q}(E)$ to be the presheaf

$$
U \mapsto\left(\pi_{p, q}(E)\right)(U)=\operatorname{colim}_{n} \operatorname{Hom}_{\mathcal{S H}} \mathcal{S}^{1}(S)\left(\Sigma_{S}^{p-2 q} \Sigma_{T}^{q+n} \Sigma_{S}^{\infty} U_{+}, E_{n}\right) .
$$

We call a morphism $f: E \rightarrow F$ in $\mathbf{S p t}_{(s, \mathfrak{p})}(S)$ a stable motivic weak-equivalence if the induced morphism $f_{*}: \pi_{p, q}(E) \rightarrow \pi_{p, q}(F)$ of presheaves is a stalkwise isomorphism of groups on $\left(\mathbf{S m}_{S}\right)_{\mathrm{Nis}}$. We often drop the word stable for simplicity. There is a closed model structure on $\operatorname{Spt}_{(s, \mathfrak{p})}(S)$ (cf Hovey [15, Section 3] and [23, Section 8.2]), whose weak-equivalences are stable motivic weak-equivalences, called the stable motivic model structure. By [15, Proposition 1.14], this model structure is obtained as a Bousfield localization of the levelwise model structure on $\operatorname{Spt}_{(s, \mathfrak{p})}(S)$ in which weak-equivalences (fibrations) are $T$-levelwise $S^{1}$-stable motivic weak-equivalences (motivic fibrations) in $\operatorname{Spt}(S)$, and $E \rightarrow F$ is a cofibration if the maps $E_{0} \rightarrow F_{0}$ and $E_{n+1} \amalg_{\Sigma_{T} E_{n}} \Sigma_{T} F_{n} \rightarrow F_{n+1}$ are cofibrations in the $S^{1}$-stable motivic model structure on $\operatorname{Spt}(S)$ for $n \geq 0$. This model structure on $\operatorname{Spt}_{(s, \mathfrak{p})}(S)$ is proper and simplicial. By [15, Theorem 3.4], we know $E \in \mathbf{S p t}_{(s, \mathfrak{p})}(S)$ is stable motivic fibrant if and only if each $E_{n}$ is $S^{1}$-stable motivic fibrant and the maps $E_{n} \rightarrow \Omega_{T} E_{n+1}$ are $S^{1}$-stable motivic weak-equivalences for $n \geq 0$. 
2.3.2 $T$-spectra model A $T$-spectrum $E$ over $S$ is a collection $\left(E_{0}, E_{1}, \ldots\right)$, $E_{i} \in \mathbf{S p c}_{\bullet}(S)$, with the maps $\Sigma_{T} E_{n} \rightarrow E_{n+1}$. They form the category $\operatorname{Spt}_{T}(S)$. For $p, q \in \mathbb{Z}$, define the presheaf $\pi_{p, q}(E)$ on $\mathbf{S m}_{S}$ by

$$
U \mapsto\left(\pi_{p, q}(E)\right)(U)=\operatorname{colim}_{n} \operatorname{Hom}_{\mathcal{H}}(S)\left(\Sigma_{S}^{p-2 q} \Sigma_{T}^{q+n} U_{+}, E_{n}\right) .
$$

There is a proper simplicial closed model structure on $\operatorname{Spt}_{T}(S)$ in which $E \rightarrow F$ is a weak-equivalence if the induced map $f_{*}: \pi_{p, q}(E) \rightarrow \pi_{p, q}(F)$ is a stalkwise isomorphism of groups on $\left(\mathbf{S m}_{S}\right)_{\mathrm{Nis}}$. This model structure is obtained as a Bousfield localization of the model structure on $\operatorname{Spt}_{T}(S)$ where weak-equivalences (fibrations) are levelwise motivic weak-equivalences (motivic fibrations) in $\mathbf{S p c}_{\bullet}(S)$. Given a motivic spectrum $E$, we let $\Omega_{s}^{\infty} E:=\operatorname{colim}_{m} \Omega_{s}^{m} E_{m}$. For a $T$-spectrum $E$, let $\Omega_{T}^{\infty} E:=$ $\operatorname{colim}_{m} \Omega_{T}^{m} E_{m}$. A $T$-spectrum $E=\left(E_{0}, E_{1}, \ldots\right)$ defines an $(s, \mathfrak{p})$-bispectrum $\mathcal{E}:=\left(\Sigma_{s}^{\infty} E_{0}, \Sigma_{s}^{\infty} E_{1}, \ldots\right)$ by taking the levelwise simplicial infinite suspensions. Conversely, given an $(s, \mathfrak{p})$-bispectrum $\mathcal{F}=\left(F_{0}, F_{1}, \ldots\right)$, we obtain a $T$-spectrum $F=\left(\Omega_{s}^{\infty} F_{0}, \Omega_{s}^{\infty} F_{1}, \ldots\right)$. The correspondence $\Sigma_{s}^{\infty}: \operatorname{Spt}_{T}(S) \leftrightarrow \operatorname{Spt}_{(s, \mathfrak{p})}(S): \Omega_{s}^{\infty}$ induces an equivalence between the homotopy categories of $\mathbf{S p t}_{T}(S)$ and $\mathbf{S p t}_{(s, \mathfrak{p})}(S)$. We write $\mathcal{S H}(S)$ for the common homotopy category. For $X \in \mathbf{S p c}_{\bullet}(S)$, one associates the infinite $T$-suspension spectrum, defined by $\Sigma_{T}^{\infty} X:=\left(X, \Sigma_{T} X, \Sigma_{T}^{2} X, \ldots\right)$, with the identity bonding maps

$$
T \wedge T^{\wedge(n-1)} \wedge X \rightarrow T^{n} \wedge X
$$

We have suspension operations $\Sigma_{T}, \Sigma_{S}, \Sigma_{t}$ to $\operatorname{Spt}_{(s, \mathfrak{p})}(S)$ and $\mathbf{S p t}_{T}(S)$. The category $\mathcal{S H}(S)$ is triangulated with the shift functor $E \mapsto E[1]$ given by $\Sigma_{S}$, and all functors $\Sigma_{T}, \Sigma_{S}, \Sigma_{t}$ are autoequivalences. For $E, F \in \operatorname{Spt}_{(s, \mathfrak{p})}(S)$, let $[E, F]_{\mathbb{A}^{1}}:=$ $\operatorname{Hom}_{\mathcal{S H}(S)}(E, F)$. There is a Quillen pair $\Sigma_{T}^{\infty}: \mathbf{S p t}(S) \leftrightarrow \mathbf{S p t}_{(s, \mathfrak{p})}(S): \Omega_{T}^{\infty}$ given by $\Sigma_{S}^{\infty}(E)=\left(E, \Sigma_{T} E, \Sigma_{T}^{2} E, \ldots\right)$ and $\Omega_{T}^{\infty}(F)=\left(\Omega_{T}^{\infty} F_{0, *}, \Omega_{T}^{\infty} F_{1, *}, \ldots\right)$. This yields an adjoint pair of derived functors

$$
\Sigma_{T}^{\infty}: \mathcal{S H}_{S^{1}}(S) \leftrightarrow \mathcal{S H}(S): R \Omega_{T}^{\infty} .
$$

For $F \in \mathbf{S p t}_{(s, \mathfrak{p})}(S)$, one has $\boldsymbol{R} \Omega_{T}^{\infty}(F)=\Omega_{T}^{\infty}(\widetilde{F})=\widetilde{F}_{0}$, where $F \rightarrow \widetilde{F}$ is a stable motivic fibrant replacement of $F$, and $\widetilde{F}_{0} \in \mathbf{S p t}(S)$ is given by $\left(\widetilde{F}_{0,0}, \widetilde{F}_{1,0}, \ldots\right)$.

2.3.3 Cohomology theories Given $E, F \in \mathcal{S H}(S)$, the $E$-cohomology of $F$ is defined by $E^{a, b}(F):=\left[F, \Sigma^{a, b} E\right]_{\mathbb{A}^{1}}$, where $a, b \in \mathbb{Z}, \Sigma^{a, b} E:=\Sigma_{s}^{a-b} \Sigma_{t}^{b} E$. For $X \in \mathbf{S m}_{S}$, using the object $\Sigma_{T}^{\infty} X_{+} \in \mathcal{S H}(S)$ we define

$$
E^{a, b}(X):=E^{a, b}\left(\Sigma_{T}^{\infty} X_{+}\right)=\left[\Sigma_{T}^{\infty} X_{+}, \Sigma^{a, b} E\right]_{\mathbb{A}^{1}}=\left[\Sigma_{T}^{\infty} \Sigma_{s}^{\infty} X_{+}, \Sigma_{s}^{a-2 b} \Sigma_{T}^{b} E\right]_{\mathbb{A}^{1}} .
$$




\section{Motivic descent for $\mathbb{A}^{1}-B G$ presheaves}

Recall that a presheaf $E$ on $\mathbf{S m}_{S}$ of objects in $\mathbf{S p c}, \mathbf{S p c}$. or $\mathbf{S p t}$ has the BG property if $E$ turns every Nisnevich square (see [32, Definition 3.1.5]) in $\mathbf{S m}_{S}$,

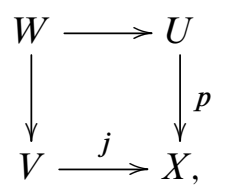

into a homotopy Cartesian square in Spc, Spc. or Spt. Recall the following, which gives a necessary and sufficient condition for a Nisnevich fibrant replacement to be an objectwise weak-equivalence; see [32, Proposition 3.1.16; 18, Theorem 1.3, Corollary 1.4].

Theorem 3.0.1 (Nisnevich descent theorem) A presheaf $E$ on $\mathbf{S m}_{S}$ of objects in Spc, Spc. or Spt is BG if and only if every Nisnevich fibrant replacement $E \rightarrow F$ is an objectwise weak-equivalence. A Nisnevich local weak-equivalence $E \rightarrow F$ of $B G$ presheaves of objects in $\mathbf{S p c}, \mathbf{S p c}$. or $\mathbf{S p t}$ is an objectwise weak-equivalence.

\subsection{Motivic descent theorem}

We establish a necessary and sufficient condition for a motivic fibrant replacement to be an objectwise weak-equivalence. Recall the following notion from Morel [31, Definition A.5]:

Definition 3.1.1 Let $E$ be a presheaf on $\mathbf{S m}_{S}$ of objects in Spc, Spc. or Spt. We say $E$ is $\mathbb{A}^{1}$-weak-invariant if the map $E(X) \rightarrow E\left(X \times \mathbb{A}^{1}\right)$ induced by the projection is a weak-equivalence for all $X \in \mathbf{S m}_{S}$. We say $E$ is $\mathbb{A}^{1}-B G$ if it is BG and $\mathbb{A}^{1}$-weakinvariant. We say $E$ is quasifibrant (resp. motivic quasifibrant) if every Nisnevich fibrant (resp. motivic fibrant) replacement $E \rightarrow F$ of $E$ is an objectwise weakequivalence.

Theorem 3.0.1 says $E$ is BG if and only if $E$ is quasifibrant. Let us begin with:

Lemma 3.1.2 Let $X \in \mathbf{S m}_{S}$.

(1) If $F$ in $\mathbf{S p c}_{\bullet}(S)(\operatorname{resp} . \mathbf{S p t}(S))$ is Nisnevich fibrant, then we have a bijection $\left[S^{p} \wedge X_{+}, F\right]_{\mathrm{Nis}} \simeq \pi_{p}(F(X))\left(\operatorname{resp} .\left[\Sigma_{S}^{\infty} X_{+}[p], F\right]_{\mathrm{Nis}} \simeq \pi_{p}(F(X))\right)$.

(2) If $F$ in $\mathbf{S p c}_{\bullet}(S)(\operatorname{resp} . \mathbf{S p t}(S))$ is motivic fibrant, then we have a bijection $\left[S^{p} \wedge X_{+}, F\right]_{\mathbb{A}^{1}} \simeq \pi_{p}(F(X))\left(\operatorname{resp} .\left[\Sigma_{S}^{\infty} X_{+}[p], F\right]_{\mathbb{A}^{1}} \simeq \pi_{p}(F(X))\right)$. 
Proof For $X \in \mathbf{S m}_{S}$, the functors $\operatorname{Ev}_{X}: \mathbf{S p c}_{\bullet}(S) \leftrightarrow \mathbf{S p c}_{\bullet}: \operatorname{sm}_{X}$ given by $\left(\operatorname{Ev}_{X}: F \mapsto\right.$ $F(X))$ and $\left(\operatorname{sm}_{X}: K \mapsto K \wedge X_{+}\right)$form a Quillen pair with respect to the Nisnevich local injective model structure and motivic model structure on Spc. $(S)$. In particular, their derived functors induce an adjoint pair of functors on the homotopy categories. The first isomorphism of (1) follows immediately from this if $F \in \mathbf{S p c}_{\mathbf{\bullet}}(S)$ is Nisnevich fibrant and the first isomorphism of (2) follows if $F \in \mathbf{S p c}_{\bullet}(S)$ is motivic fibrant. The second isomorphisms of (1) and (2) follow from the first set of isomorphisms by applying Theorem 2.2.1 and (2.2.1).

The following result follows immediately from Theorem 3.0.1 and [30, Lemma 4.1.4].

Lemma 3.1.3 Let $E$ be a $B G$ presheaf on $\mathbf{S m}_{S}$ of objects in Spc, Spc. or Spt.

(1) Let $E \rightarrow E^{\prime}$ be a Nisnevich fibrant replacement. Then $E$ is $\mathbb{A}^{1}$-weak-invariant if and only if so is $E^{\prime}$.

(2) $E$ is $\mathbb{A}^{1}$-weak-invariant if and only if $E$ is $\mathbb{A}^{1}$-local.

Lemma 3.1.4 A motivic fibrant replacement of an $\mathbb{A}^{1}-B G$ presheaf on $\mathbf{S m}_{S}$ of objects in Spc, Spc. or Spt is also a Nisnevich fibrant replacement.

Proof We consider the case of presheaves of spectra as the other cases are similar. Let $f: E \rightarrow F$ be a motivic fibrant replacement. Since $F$ is Nisnevich fibrant and since cofibrations in the motivic model structure are Nisnevich cofibrations, it suffices to show that $f$ is a Nisnevich local weak-equivalence. Factor $f$ as a composition $f^{\prime} \circ g: E \rightarrow E^{\prime} \rightarrow F$, where $g$ is a Nisnevich trivial cofibration (thus a motivic trivial cofibration) and $f^{\prime}$ is a Nisnevich fibration. By the two-out-of-three axiom, $f^{\prime}$ is a motivic weak-equivalence. We need to show that $f^{\prime}$ is a Nisnevich local weakequivalence. Since $F$ is Nisnevich fibrant and $f^{\prime}$ is a Nisnevich fibration, it follows that $E^{\prime}$ is Nisnevich fibrant. Thus, $g$ defines a Nisnevich fibrant replacement of $E$. Moreover, by Lemma 3.1.3, we see that $E^{\prime}$ is $\mathbb{A}^{1}$-local. Hence $E^{\prime}$ is motivic fibrant. Now by Lemma 3.1.2, $f^{\prime}: E^{\prime} \rightarrow F$ is an objectwise weak-equivalence, thus a Nisnevich local weak-equivalence.

Theorem 3.1.5 (Motivic descent theorem) Let $E$ be a presheaf on $\mathbf{S m}_{S}$ of objects in Spc, Spc. or Spt. Then $E$ is $\mathbb{A}^{1}-B G$ if and only if it is motivic quasifibrant. A motivic weak-equivalence of $\mathbb{A}^{1}-B G$ presheaves is an objectwise weak-equivalence.

Proof Suppose that $E$ is motivic quasifibrant. Let $f: E \rightarrow E^{\prime}$ be a motivic fibrant replacement. Then $E^{\prime}$ is Nisnevich fibrant (thus BG) and $\mathbb{A}^{1}$-local. So, by 
Lemma 3.1.3, $E^{\prime}$ is $\mathbb{A}^{1}$-BG Since $E$ is motivic quasifibrant, $f$ is an objectwise weakequivalence, thus a Nisnevich local weak-equivalence. So, by Theorem 3.0.1, $E$ is BG, and by Lemma 3.1.3, it is $\mathbb{A}^{1}$-weak-invariant, that is, $E$ is $\mathbb{A}^{1}-\mathrm{BG}$. Conversely, suppose $E$ is an $\mathbb{A}^{1}$-BG Let $f: E \rightarrow E^{\prime}$ be a motivic fibrant replacement. By Lemma 3.1.4, $f$ is also a Nisnevich fibrant replacement. That $f$ is an objectwise weak-equivalence follows now from Theorem 3.0.1. Thus $E$ is motivic quasifibrant. This proves the first assertion. To prove the second one, given a motivic weak-equivalence $f: E \rightarrow F$ of $\mathbb{A}^{1}-\mathrm{BG}$ presheaves, form a commutative diagram

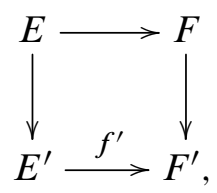

where the vertical arrows are motivic fibrant replacements, which are objectwise weak-equivalences by the first part. By the two-out-of-three axiom, $f^{\prime}$ is a motivic weak-equivalence. In this case, we have shown in the proof of Lemma 3.1.4 that $f^{\prime}$ is an objectwise weak-equivalence. But, we saw that two vertical arrows are also objectwise weak-equivalences. Thus, $f$ is an objectwise weak-equivalence.

Corollary 3.1.6 The isomorphisms in Lemma 3.1.2(1) hold for all BG pointed motivic spaces and spectra, while Lemma 3.1.2(2) holds for all $\mathbb{A}^{1}-B G$ ones.

Corollary 3.1.7 The class of motivic quasifibrant presheaves on $\mathbf{S m}_{S}$ of objects in Spc, Spc. or Spt is closed under taking filtered colimits.

Proof This follows by Theorem 3.1.5 and the proof of [30, Corollary 4.2.7].

For $E=\left(E_{0}, E_{1}, \ldots\right) \in \operatorname{Spt}(S)$, let $E\{n\}$ denote the motivic spectrum $\left(E_{n}, E_{n+1}, \ldots\right)$. Let $m \geq-1$. We say that $E$ is an objectwise (resp. motivic) $\Omega_{s}-$ spectrum above level $m$ if the map $E_{n} \rightarrow \Omega_{s} E_{n+1}$ is an objectwise (resp. motivic) weak-equivalence for each $n>m$. An objectwise (resp. motivic) $\Omega_{s}$-spectrum above level $m=-1$ will be called an objectwise (resp. motivic) $\Omega_{s}$-spectrum.

Corollary 3.1.8 Suppose $E \in \operatorname{Spt}(S)$ is $\mathbb{A}^{1}-B G$ Let $E \rightarrow F$ be a motivic fibrant replacement.

(1) For each $m, n, p \geq 0$, the map $\Omega_{s}^{m} F_{n} \rightarrow \Omega_{s}^{m+p} F_{n+p}$ is an objectwise weakequivalence.

(2) For each $m, n \geq 0$, the motivic spectrum $\Omega_{s}^{m} F\{n\}$ is $S^{1}$-stable motivic fibrant.

(3) For each $n>m$, the map $E_{n} \rightarrow F_{n}$ is an objectwise weak-equivalence if $E$ is an objectwise $\Omega_{s}$-spectrum above level $m$. 
Proof A motivic spectrum is $S^{1}$-stable motivic fibrant if and only if it is levelwise motivic fibrant and a motivic $\Omega_{s}$-spectrum. Thus, each $F_{n} \in \mathbf{S p c}_{\mathbf{\bullet}}(S)$ is motivic fibrant. Since $S^{1}$ is cofibrant, each $\Omega_{s}^{m} F_{n}$ is also motivic fibrant and the map $F_{n} \rightarrow \Omega_{s} F_{n+1}$ is a motivic weak-equivalence. In particular, the map $\boldsymbol{R} \Omega_{s}^{m} F_{n} \rightarrow \boldsymbol{R} \Omega_{s}^{m+p} F_{n+p}$ is a motivic isomorphism. Since each $\Omega_{s}^{m} F_{n}$ is motivic fibrant, each map $\Omega_{s}^{m} F_{n} \rightarrow$ $\Omega_{s}^{m+p} F_{n+p}$ is a motivic weak-equivalence for $m, n, p \geq 0$. By Lemma 3.1.2, this map is an objectwise weak-equivalence, proving (1). Since each $\Omega_{s}^{m} F_{n}$ is motivic fibrant and the map $\Omega_{s}^{m} F_{n+p} \rightarrow \Omega_{s}^{m+1} F_{n+p+1}$ is a motivic weak-equivalence, it follows that $\Omega_{s}^{m} F\{n\}$ is $S^{1}$-stable motivic fibrant, proving (2). For (3), we first apply Theorem 3.1.5 to deduce that $E \rightarrow F$ is an objectwise stable weak-equivalence. For $n>m, p \geq 0$ and $X \in \mathbf{S m}_{S}$, we get isomorphisms

$$
\begin{aligned}
\pi_{p}\left(E_{n}(X)\right) \simeq{ }^{1} \operatorname{colim}_{q} \pi_{p+q}\left(E_{n+q}(X)\right) \simeq \pi_{p-n}(E(X)) & \simeq \pi_{p-n}(F(X)) \\
& \simeq^{2} \pi_{p}\left(F_{n}(X)\right),
\end{aligned}
$$

where $\simeq^{1}$ holds because $E$ is an objectwise $\Omega_{s}$-spectrum above level $m$, and $\simeq^{2}$ holds because $F$ is an objectwise $\Omega_{s}$-spectrum.

\section{2 $\mathbb{A}^{1}-B G$ property of motivic spaces and motivic spectra}

We study the $\mathbb{A}^{1}-B G$ property of $E \in \mathbf{S p t}(\mathbb{C})$ in terms of the property of its levels. Given any $E \in \mathbf{S p c}_{\bullet}(S), K \in \mathbf{S p c}_{\bullet}$ and $U \in \mathbf{S m}_{S}$, there is an isomorphism $\mathcal{H o m}_{\bullet}(K, E)(U) \simeq \operatorname{Hom}_{\bullet}(K, E(U))$ in $\mathbf{S p c}_{\bullet}$. Thus, we have the isomorphism $\left(\Omega_{s} E\right)(U) \simeq \Omega_{s}(E(U))$. Since $\operatorname{Hom}_{\bullet}\left(S^{1},-\right)$ preserves weak-equivalences and fibration sequences in $\mathbf{S p c}_{\bullet}$, we deduce:

Corollary 3.2.1 The functor $\Omega_{s}(-)$ preserves objectwise weak-equivalences, $B G$ property and $\mathbb{A}^{1}$-weak-invariance of $\mathbf{S p c}_{\bullet}(S)$. It preserves motivic weak-equivalences of $\mathbb{A}^{1}-B G$ pointed motivic spaces. If $E \in \mathbf{S p c}_{\bullet}(\mathbb{C})$ is $\mathbb{A}^{1}-B G$, the natural map $\Omega_{s} E \rightarrow \boldsymbol{R} \Omega_{s} E$ is an isomorphism in $\mathcal{H}_{\bullet}(S)$.

Proof The first statement is obvious. The second one follows from the first and Theorem 3.1.5. To see the last one, take a motivic fibrant replacement $E \rightarrow E^{\prime}$, apply the second one, and use the isomorphism $\Omega_{s} E^{\prime} \simeq R \Omega_{s} E^{\prime}$.

We say $E \in \operatorname{Spt}(S)$ is levelwise $\mathbb{A}^{1}-B G$ if each $E_{n}$ is $\mathbb{A}^{1}-\mathrm{BG}$.

Corollary 3.2.2 Let $E \rightarrow F$ be a levelwise motivic weak-equivalence of levelwise $\mathbb{A}^{1}-B G$ motivic spectra. If $E$ is a motivic $\Omega_{s}$-spectrum, then so is $F$.

Proof This is an immediate consequence of Theorem 3.1.5 and Corollary 3.2.1. 
Lemma 3.2.3 Let $f: E \rightarrow F$ be a morphism of levelwise $\mathbb{A}^{1}-B G$ motivic $\Omega_{s}-$ spectra on $\mathbf{S m}_{S}$. Then $f$ is an $S^{1}$-stable motivic weak-equivalence if and only if each $f_{n}: E_{n} \rightarrow F_{n}$ is an objectwise weak-equivalence.

Proof Suppose that $f: E \rightarrow F$ is an $S^{1}$-stable motivic weak-equivalence. Let $n, p \geq 0$ and $U \in \mathbf{S m}_{S}$. Since $E$ and $F$ are levelwise $\mathbb{A}^{1}-$ BG, by Corollary 3.1.6,

$$
\begin{aligned}
\pi_{p}\left(E_{n}(U)\right) \simeq\left[S^{p} \wedge U_{+}, E_{n}\right]_{\mathbb{A}^{1}} & \simeq{ }^{1}\left[S^{p} \wedge U_{+}, \Omega_{s}^{m-n} E_{m}\right]_{\mathbb{A}^{1}} \\
& \simeq^{2}\left[S^{p} \wedge U_{+}, \boldsymbol{R} \Omega_{s}^{m-n} E_{m}\right]_{\mathbb{A}^{1}} \\
& \simeq{ }^{3}\left[S^{m+p-n} \wedge U_{+}, E_{m}\right]_{\mathbb{A}^{1}},
\end{aligned}
$$

where $\simeq^{1}$ holds because $E$ is a motivic $\Omega_{s}$-spectrum, $\simeq^{2}$ holds by Corollary 3.2.1 and $\simeq{ }^{3}$ holds by the adjointness. But $m \gg 0$ is arbitrary so $\left[S^{m+p-n} \wedge U_{+}, E_{m}\right]_{\mathbb{A}^{1}}=$ $\operatorname{colim}_{m}\left[S^{m+p-n} \wedge U_{+}, E_{m}\right]_{\mathbb{A}^{1}}$, which is $\left[\Sigma_{S}^{\infty} U_{+}[p-n], E\right]_{\mathbb{A}^{1}}$ by (2.2.1). Similarly, $\pi_{p}\left(F_{n}(U)\right) \simeq\left[\Sigma_{s}^{\infty} U_{+}[p-n], F\right]_{\mathbb{A}^{1}}$. Since $f$ is an $S^{1}$-stable motivic weakequivalence, we deduce that the map $f_{n}: E_{n} \rightarrow F_{n}$ is an objectwise weak-equivalence. The other direction is obvious.

Corollary 3.2.4 Every levelwise $\mathbb{A}^{1}-B G$ motivic $\Omega_{s}$-spectrum is motivic quasifibrant.

Proof Consider an $S^{1}$-stable motivic fibrant replacement of the given one. Since an $S^{1}$-stable motivic fibrant motivic spectrum is a levelwise motivic fibrant (thus $\mathbb{A}^{1}-\mathrm{BG}$ ) motivic $\Omega_{s}$-spectrum, this corollary holds by Lemma 3.2.3 and Theorem 3.1.5.

\subsection{Motivic descent for $(s, \mathfrak{p})$-bispectra}

Given an open or a closed immersion of schemes $A \subseteq B$ in $\mathbf{S m}_{S}$, let $\Omega_{B / A}(-)$ be the functor $E \mapsto \Omega_{B / A} E=\left(\Omega_{B / A} E_{0}, \Omega_{B / A} E_{1}, \ldots\right)$ on $\operatorname{Spt}(S)$, where $\Omega_{B / A} F=$ $\mathcal{H}_{\circ}(B / A, F)$ is the objectwise fiber of the map $\mathcal{H o m}(B, F) \rightarrow \mathcal{H o m}(A, F)$ for $F \in$ Spc. $_{\bullet}(S)$; see [18, Corollary 1.10]. There is an objectwise fiber sequence of presheaves $\Omega_{B / A} E \rightarrow E_{B} \rightarrow E_{A}$, where $E_{B}(X):=E(B \times X)=\mathcal{H o m}(B, E)(X)$. Recall (see [18, Corollary 3.2]) that given an objectwise fiber sequence as above, the map $E_{B} /\left(\Omega_{B / A} E\right) \rightarrow E_{A}$ is an objectwise $S^{1}$-stable weak-equivalence. The natural isomorphism $S^{1} \wedge E_{X} \rightarrow\left(S^{1} \wedge E\right)_{X}$, for $E \in \mathbf{S p c}_{\bullet}(S)$ and $X \in \mathbf{S m}_{S}$, and the above cofiber sequence, give a natural map $S^{1} \wedge \Omega_{B / A} E_{n} \rightarrow \Omega_{B / A}\left(S^{1} \wedge E_{n}\right)$ for $E \in \operatorname{Spt}(S)$. Composed with the bonding map $\Omega_{B / A}\left(S^{1} \wedge E_{n}\right) \rightarrow \Omega_{B / A}\left(E_{n+1}\right)$, we see that $E \rightarrow \Omega_{B / A} E$ is an endofunctor on $\operatorname{Spt}(S)$. There is a natural bijection $\operatorname{Hom}_{\operatorname{Spt}(S)}\left(\Sigma_{B / A} E, F\right) \simeq \operatorname{Hom}_{\operatorname{Spt}(S)}\left(E, \Omega_{B / A} F\right)$. The following analogue of Corollary 3.2.1 for motivic spectra is immediate from Theorem 3.1.5 and the above objectwise cofiber sequence. 
Lemma 3.3.1 The functor $\Omega_{B / A}(-)$ preserves objectwise weak-equivalences, $B G$ property and $\mathbb{A}^{1}$-weak-invariance of motivic spectra. It preserves motivic weakequivalences of $\mathbb{A}^{1}-B G$ motivic spectra. If $E \in \operatorname{Spt}(S)$ is $\mathbb{A}^{1}-B G$, then the natural map $\Omega_{B / A} E \rightarrow \boldsymbol{R} \Omega_{B / A} E$ is an isomorphism in $\mathcal{S} \mathcal{H}_{S^{1}}(S)$. If $f: E \rightarrow F$ is an $S^{1}$ stable motivic weak-equivalence of $\mathbb{A}^{1}-B G$ motivic spectra, then $\Omega_{B / A} f: \Omega_{B / A} E \rightarrow$ $\Omega_{B / A} F$ is also an $S^{1}$-stable motivic weak-equivalence.

Recall from Sections 2.3.1 and 2.3.2 that an $(s, \mathfrak{p})$-bispectrum $E=\left(E_{m, n}\right)_{m, n \geq 0}$ gives a sequence $\left(E_{0}, E_{1}, \ldots\right)$ of motivic spectra with bonding maps $\Sigma_{T} E_{n}=$ $T \wedge E_{n} \rightarrow E_{n+1}$.

Definition 3.3.2 For $E \in \mathbf{S p t}_{(s, \mathfrak{p})}(S)$, we say that $E$ is a motivic $\Omega_{T}$-bispectrum if the adjoint maps $E_{n} \rightarrow \Omega_{T} E_{n+1}$ are motivic weak-equivalences in $\operatorname{Spt}(S)$ for $n \geq 0$. We say that $E$ is $\mathbb{A}^{1}-B G$ if each $E_{n}$ is an $\mathbb{A}^{1}-\mathrm{BG}$ motivic spectrum for $n \geq 0$.

Theorem 3.3.3 Let $f: E \rightarrow F$ be a stable motivic weak-equivalence of $\mathbb{A}^{1}-B G$ motivic $\Omega_{T}$-bispectra on $\mathbf{S m}_{S}$. Then $f$ is a T-levelwise objectwise weak-equivalence, ie each $f_{n}: E_{n} \rightarrow F_{n}$ is an objectwise weak-equivalence.

Proof Let $n \geq 0, p \in \mathbb{Z}$ and $U \in \mathbf{S m}_{S}$. Since $E$ is ( $T$-levelwise) $\mathbb{A}^{1}-\mathrm{BG}$, apply Corollary 3.1.6 to get

$$
\pi_{p}\left(E_{n}\right)(U) \simeq\left[\Sigma_{s}^{\infty} U_{+}[p], E_{n}\right]_{\mathbb{A}^{1}} \simeq^{1}\left[\Sigma_{s}^{\infty} U_{+}[p], \Omega_{T}^{m-n} E_{m}\right]_{\mathbb{A}^{1}},
$$

where $\simeq^{1}$ holds for $E$ is a motivic $\Omega_{T}$-bispectrum. By Lemma 3.3.1, this equals $\left[\Sigma_{S}^{\infty} U_{+}[p], \boldsymbol{R} \Omega_{T}^{m-n} E_{m}\right]_{\mathbb{A}^{1}}$. By adjointness it equals $\left[\Sigma_{T}^{m-n} \Sigma_{s}^{p} \Sigma_{S}^{\infty} U_{+}, E_{m}\right]_{\mathbb{A}^{1}}$. Since $m \gg 0$ is arbitrary,

$$
\left[\Sigma_{T}^{m-n} \Sigma_{S}^{p} \Sigma_{s}^{\infty} U_{+}, E_{m}\right]_{\mathbb{A}^{1}}=\operatorname{colim}_{m}\left[\Sigma_{T}^{m-n} \Sigma_{s}^{p} \Sigma_{s}^{\infty} U_{+}, E_{m}\right]_{\mathbb{A}^{1}},
$$

which is $\pi_{p-n,-n}(E)(U)$ by definition in Section 2.3. Similarly, $\pi_{p}\left(F_{n}(U)\right) \simeq$ $\pi_{p-n,-n}(F)(U)$. Now, by our assumptions, the map $\pi_{p}\left(E_{n}\right) \rightarrow \pi_{p}\left(F_{n}\right)$ induces an isomorphism of the associated Nisnevich sheaves so that $f_{n}: E_{n} \rightarrow F_{n}$ is a Nisnevich local weak-equivalence, and hence an $S^{1}$-stable motivic weak-equivalence. Since these are $\mathbb{A}^{1}-\mathrm{BG}$ motivic spectra, by Theorem 3.1.5 each $f_{n}$ is an objectwise weakequivalence.

Corollary 3.3.4 For $E \in \mathbf{S p t}_{(s, \mathfrak{p})}(S)$, let $f: E \rightarrow E^{\prime}$ be a stable motivic fibrant replacement. Then $E$ is an $\mathbb{A}^{1}-B G$ motivic $\Omega_{T}$-bispectrum if and only if $f$ is a $T$-levelwise objectwise weak-equivalence. 
Proof The forward direction is obvious by Theorem 3.3.3. For the backward direction, note that each level $E_{n} \rightarrow E_{n}^{\prime}$ is an objectwise weak-equivalence, with $E_{n}^{\prime}$ is motivic fibrant, so that each $E_{n}$ is $\mathbb{A}^{1}-\mathrm{BG}$ by Theorem 3.1.5. It only remains to see that $E$ is a motivic $\Omega_{T}$-bispectrum. This follows from Lemma 3.3.1.

\section{Singular semitopologization}

\subsection{Definition and basic properties}

From now, we take $S=\operatorname{Spec}(\mathbb{C})$. For a complex algebraic variety $U$, let $U^{\text {an }}$ be its associated complex analytic space. We recall the semitopologization of Friedlander and Walker from [11, Definition 10].

4.1.1 Realization and diagonal of a simplicial spectrum We briefly review the diagonal and the realization of a simplicial spectrum. For a bisimplicial set $A_{* *}$, the realization $|A|$ is the simplicial set obtained by taking the coequalizer of the diagram $\bigsqcup_{(\alpha:[n] \rightarrow[k]) \in \Delta^{\text {op }}} A_{n} \times \Delta[k] \rightrightarrows \bigsqcup_{n \geq 0} A_{n} \times \Delta[n]$, where the two morphisms are $(\alpha, x, t) \mapsto\left(x, \alpha^{*}(t)\right)$ and $(\alpha, x, t) \mapsto\left(\alpha_{*}(x), t\right)$. If $A_{* *}$ is a simplicial object in Spc. then $|A|$ is obtained by replacing $A_{n} \times \Delta[k]$ by $A_{n} \wedge(\Delta[k])_{+}$in the above. The diagonal $\operatorname{diag} A$ is the composite $A_{* *} \circ \delta: \Delta^{\mathrm{op}} \rightarrow \Delta^{\mathrm{op}} \times \Delta^{\mathrm{op}} \rightarrow$ Set. There is a natural isomorphism $\operatorname{diag} A \rightarrow|A|$; see [2, Proposition B.1]. If $E: \Delta^{\mathrm{op}} \rightarrow$ Spt is a simplicial spectrum, its realization $|E|$ is defined as above, where $A_{n} \times \Delta[k]$ is replaced by $E(\Delta[n]) \wedge(\Delta[k])_{+}$. A simplicial spectrum $E$ can be seen as a sequence $\left(E_{* *}^{0}, E_{* *}^{1}, \ldots\right)$, where each $E_{* *}^{n}$ is a pointed bisimplicial set, with the bonding maps $S^{1} \wedge E_{* *}^{n} \rightarrow E_{* *}^{n+1}$. So the spectrum $|E|$ has $|E|_{n}=\left|E_{* *}^{n}\right|$ in Spc $\mathbf{S p}_{\bullet}$, with the bonding maps $S^{1} \wedge\left|E_{* *}^{n}\right| \rightarrow\left|E_{* *}^{n+1}\right|$. The diagonal diag $E$ of $E$ is the spectrum with $(\operatorname{diag} E)_{n}=\operatorname{diag}\left(E_{* *}^{n}\right)$. We have $S^{1} \wedge E_{p}^{n} \rightarrow E_{p}^{n+1}$, where $E_{p}^{n}=(E(\Delta[p]))_{n}$ or the map of pointed sets $\left(S^{1}\right)_{i} \wedge E_{p, i}^{n} \rightarrow E_{p, i}^{n+1}$. The maps $\left(S^{1}\right)_{p} \wedge E_{p, p}^{n} \rightarrow E_{p, p}^{n+1}$ give the bonding maps $S^{1} \wedge(\operatorname{diag} E)_{n} \rightarrow(\operatorname{diag} E)_{n+1}$ of the spectrum $\operatorname{diag} E$. From the case of bisimplicial sets, one gets $\operatorname{diag} E \simeq|E|$. If $E$ is a presheaf of simplicial spectra on $\mathbf{S c h}_{S}$ or $\mathbf{S m}_{S}$, we define $|E|$ and $\operatorname{diag} E$ objectwise. Thus, for a simplicial presheaf of spectra $E$ on $\mathbf{S c h}_{S}$ or $\mathbf{S m}_{S}$, we have diag $E \simeq|E|$.

4.1.2 Semitopologization For $T \in \mathcal{T} o p$, let $\left(T \mid \operatorname{Var}_{\mathbb{C}}\right)$ be the category whose objects are $(f, U)$, where $U \in \operatorname{Var}_{\mathbb{C}}$, and $f: T \rightarrow U$ an is a continuous map. A morphism from $(f, U)$ to $(g, V)$ is a morphism $h: U \rightarrow V$ in $\operatorname{Var}_{\mathbb{C}}$ such that the map $h^{\text {an }}: U^{\text {an }} \rightarrow V^{\text {an }}$ satisfies $h^{\text {an }} \circ f=g$. Recall that $\Delta_{\top}^{\bullet}=\left\{\Delta_{\top}^{n}\right\}_{n \geq 0}$ is a cosimplicial topological space with the natural cofaces $\partial^{i}$ and the codegeneracies $s^{i}$. For $n>0$ and $0 \leq i \leq n$, define 
$\partial_{i}:\left(\Delta_{\top}^{n} \mid \operatorname{Var}_{\mathbb{C}}\right)^{\mathrm{op}} \rightarrow\left(\Delta_{\top}^{n-1} \mid \operatorname{Var}_{\mathbb{C}}\right)^{\mathrm{op}}$ by

$$
\left(f: \Delta_{\top}^{n} \rightarrow U^{\mathrm{an}}\right) \mapsto\left(f \circ \partial^{i}: \Delta_{\top}^{n-1} \rightarrow \Delta_{\top}^{n} \rightarrow U^{\mathrm{an}}\right) .
$$

For $n \geq 0$ and $0 \leq i \leq n$, define $s_{i}:\left(\Delta_{\top}^{n} \mid \operatorname{Var}_{\mathbb{C}}\right)^{\text {op }} \rightarrow\left(\Delta_{\top}^{n+1} \mid \operatorname{Var}_{\mathbb{C}}\right)^{\text {op }}$ by

$$
\left(f: \Delta_{\top}^{n} \rightarrow U^{\mathrm{an}}\right) \mapsto\left(f \circ s^{i}: \Delta_{\top}^{n+1} \rightarrow \Delta_{\top}^{n} \rightarrow U^{\mathrm{an}}\right) .
$$

Recall the following from [11]:

Definition 4.1.1 Let $E$ be a presheaf on $\mathbf{S c h}_{\mathbb{C}}$ of objects in Spc, Spc or Spt. Let $X \in \mathbf{S c h}_{\mathbb{C}}$ and let $T \in \mathcal{T}$ op. Define $E(T \times X)=\operatorname{colim}_{(f, U) \in\left(T \mid \operatorname{Var}_{\mathbb{C}}\right)^{\text {op }}} E(U \times X)$. Consider $E\left(\Delta_{\top}^{\bullet} \times X\right)=\left\{E\left(\Delta_{\top}^{n} \times X\right)\right\}_{n \geq 0}$, which is a simplicial object in Spc, Spc. or Spt. Let $E^{\text {sst }}(X):=\left|E\left(\Delta_{\top}^{\bullet} \times X\right)\right|$. This $E^{\text {sst }}$ is a presheaf on $\mathbf{S c h}_{\mathbb{C}}$ of objects in Spc, Spc. or Spt, called the semitopologization of $E$.

There is a natural morphism of presheaves $E \rightarrow E^{\text {sst }}$ on $\mathbf{S c h}_{\mathbb{C}}$, which gives a natural transformation Id $\rightarrow(-)^{\text {sst }}$ of functors on presheaves on $\mathbf{S c h}_{\mathbb{C}}$.

Lemma 4.1.2 Let $E$ be a presheaf on $\mathbf{S c h}_{\mathbb{C}}$ of objects in Spc, Spc. or Spt. Let $X \in \mathbf{S c h}_{\mathbb{C}}$. Define a presheaf on $\mathbf{S c h}_{\mathbb{C}}$ by $E_{X}(U):=E(U \times X)$. Then

$$
\left(E_{X}\right)^{\text {sst }}=\left(E^{\text {sst }}\right)_{X} \text {. }
$$

Proof For $U \in \operatorname{Sch}_{\mathbb{C}}$, we have $\left(E^{\text {sst }}\right)_{X}(U)=E^{\text {sst }}(X \times U)=\left|\left\{E\left(\Delta_{\text {top }}^{n} \times X \times U\right)\right\}_{n}\right|=$ $\left|\left\{\operatorname{colim}_{(f, C)} E(C \times X \times U)\right\}_{n}\right|=\left|\left\{\operatorname{colim}_{(f, C)} E_{X}(C \times U)\right\}_{n}\right|=\left|\left\{E_{X}\left(\Delta_{\text {top }}^{n} \times U\right)\right\}_{n}\right|$. This is by definition $\left(E_{X}\right)^{\text {sst }}(U)$.

When $E$ is a presheaf on $\mathbf{S m}_{\mathbb{C}}$, it is well known that the realization of $E\left(\Delta^{\bullet} \times-\right)$ is $\mathbb{A}^{1}$-weak-invariant (see Friedlander and Suslin [6, Proposition 7.2] and Friedlander and Voevodsky [7, page 150]). Its semitopological analogue also holds by [8, Lemma 1.2]:

Theorem 4.1.3 Let $E$ be a presheaf on $\mathbf{S c h}_{\mathbb{C}}$ of objects in Spc, Spc • or Spt. Then $E^{\text {sst }}$ is $\mathbb{A}^{1}$-weak-invariant.

\subsection{Semitopologization and $\wedge$-product}

Recall that for $A, B \in \mathbf{S p c}$. (all base points are denoted by $\star$ ), we have $A \wedge B=$ $(A \times B) /(A \vee B)$, where $A \vee B=(A \times \star) \cup(\star \times B)$ in $A \times B$. For two presheaves $E$ and $F$ on a category $\mathcal{C}$ of objects in Spc $\mathbf{s}_{\bullet}$, define the presheaf $E \wedge F$ on $\mathcal{C}$ objectwise by $(E \wedge F)(U)=E(U) \wedge F(U)$, so one still has $E \wedge F=(E \times F) /(E \vee F)$. When $F$ is a presheaf of spectra on $\operatorname{Sch}_{\mathbb{C}}$ while $E$ is as above, we define $E \wedge F$ levelwise, namely, $E \wedge F=\left(E \wedge F_{0}, E \wedge F_{1}, \ldots\right)$. 
Proposition 4.2.1 Let $E, F, F^{\prime}$ be presheaves on $\mathbf{S c h}_{\mathbb{C}}$ of objects in $\mathbf{S p c}_{\bullet}$. Then we have the following identities:

(1) $(E \times F)^{\mathbf{s s t}}=E^{\mathbf{s s t}} \times F^{\mathbf{s s t}}$.

(2) $(E \vee F)^{\text {sst }}=E^{\text {sst }} \vee F^{\text {sst }}$.

(3) If $F \subset F^{\prime}$, then $\left(F^{\prime} / F\right)^{\text {sst }}=F^{\prime \text { sst }} / F^{\text {sst }}$.

(4) $(E \wedge F)^{\text {sst }}=E^{\text {sst }} \wedge F^{\text {sst }}$.

(5) When $E$ is as above and $F$ is a presheaf of spectra on $\operatorname{Sch}_{\mathbb{C}},(E \wedge F)^{\text {sst }}=$ $E^{\text {sst }} \wedge F^{\text {sst }}$.

Proof Let $X \in \mathbf{S c h}_{\mathbb{C}}$ be a fixed scheme. For (1), let $U \in \mathbf{S c h}_{\mathbb{C}}$. Note that $(E \times F)(U \times$ $X)=E(U \times X) \times F(U \times X)$. Over the objects $\left(f: \Delta_{\top}^{n} \rightarrow U^{\text {an }}\right)$ of the filtered category $\left(\Delta_{\top}^{n} \mid \operatorname{Var}_{\mathbb{C}}\right)^{\text {op }}$, take the filtered colimit. By Mac Lane [26, Section IX.2 Theorem 1] finite limits (eg products) commute with filtered colimits, so that $(E \times F)\left(\Delta_{\top}^{n} \times X\right)=$ $E\left(\Delta_{\top}^{n} \times X\right) \times F\left(\Delta_{\top}^{n} \times X\right)$. Taking the diagonals, we obtain (1). For (2), for each $U \in \operatorname{Sch}_{\mathbb{C}}$, note that $(E \vee F)(U \times X)=\operatorname{colim}\{E(U \times X) \times \star \leftarrow \star \times \star \rightarrow \star \times F(U \times X)\}$. Take the filtered colimits over the objects $\left(f: \Delta_{\top}^{n} \rightarrow U^{\text {an }}\right)$ of $\left(\Delta_{\top}^{n} \mid \operatorname{Var}_{\mathbb{C}}\right)^{\text {op }}$. Colimits commute among themselves (see [26, Section IX.8]) so that $(E \vee F)\left(\Delta_{\top}^{n} \times X\right)=$ $E\left(\Delta_{\top}^{n} \times X\right) \vee F\left(\Delta_{\top}^{n} \times X\right)$. This implies (2), by taking the diagonals. For (3), similarly we consider instead $F^{\prime}(U \times X) / F(U \times X)=\operatorname{colim}\left\{\star \leftarrow F(U \times X) \rightarrow F^{\prime}(U \times X)\right\}$, and repeat the same procedure. This proves (3). Now, (4) follows from (1)-(3). For (5), since the limits and colimits of spectra are all defined levelwise, this part follows from (4).

\section{Semitopologization of presheaves on smooth schemes}

\subsection{Artificial extension}

We discuss how to define semitopologization on presheaves on $\mathbf{S m}_{\mathbb{C}}$. For a presheaf $E$ on $\mathbf{S c h}_{\mathbb{C}}$ of objects in Spc, Spc. or Spt, we used the categories $\left(\Delta_{\uparrow}^{n} \mid \operatorname{Var}_{\mathbb{C}}\right)^{\text {op }}$ to define $E^{\text {sst }}$ in Section 4. If $E$ is defined only on $\mathbf{S m}_{\mathbb{C}}$ a priori, then one may either extend the functor $F$ to all of $\mathbf{S c h}_{\mathbb{C}}$ or shrink the indexing categories to, say, $\left(\Delta_{\top}^{n} \mid \mathbf{S m}_{\mathbb{C}}\right)^{\text {op }}$. Both raise some issues. Extension of $F$ from $\mathbf{S m}_{\mathbb{C}}$ to $\mathbf{S c h}_{\mathbb{C}}$ is not unique. On the other hand, the inclusion $\left.\left(\Delta_{\top}^{n} \mid \mathbf{S m}_{\mathbb{C}}\right)^{\mathrm{op}}\right) \hookrightarrow\left(\Delta_{\uparrow}^{n} \mid \mathbf{V a r}_{\mathbb{C}}\right)^{\mathrm{op}}$ is not cofinal. Furthermore, the indexing categories $\left(\Delta_{T}^{n} \mid \mathbf{S m}_{\mathbb{C}}\right)^{\text {op }}$ are not filtered, over which the colimits have poor properties. To avoid these, we use a fixed functorial extension process to obtain a presheaf on $\mathbf{S c h} \mathbb{C}$, and then apply the sst-functor of Section 4 . 
Let $W \in \mathbf{S c h}_{\mathbb{C}}$. Consider the objects $(f, X)$, where $X \in \mathbf{S m}_{\mathbb{C}}$ and $f: W \rightarrow X$ is a morphism in $\mathbf{S c h}_{\mathbb{C}}$. Given $(f, X)$ and $(g, Y)$, with $X, Y \in \mathbf{S m}_{\mathbb{C}}$, a morphism $\psi$ from $(f, X)$ to $(g, Y)$ is defined to be a morphism $\psi: X \rightarrow Y$ in $\mathbf{S c h}_{\mathbb{C}}$ such that $\psi \circ f=g$. Let $\left(W \mid \mathbf{S m}_{\mathbb{C}}\right)^{\text {op }}$ be the category of the pairs $(f, X)$ with the above morphisms.

Definition 5.1.1 Let $E$ be a presheaf on $\mathbf{S m}_{\mathbb{C}}$ of objects in a cocomplete category $\mathcal{M}$. For $W \in \mathbf{S c h}_{\mathbb{C}}$, define the artificial extension $\bar{E}$ of $E$ by

$$
\bar{E}(W):=\operatorname{colim}_{(f, X) \in\left(W \mid \mathbf{S m}_{\mathbb{C}}\right)^{\text {op }}} E(X) .
$$

If $W \in \mathbf{S m}_{\mathbb{C}}$, then $\left(W \mid \mathbf{S m}_{\mathbb{C}}\right)^{\text {op }}$ has the terminal object $\left(\operatorname{Id}_{W}, W\right)$ so $\bar{E}(W)=E(W)$. One checks that given $\phi: W \rightarrow W^{\prime}$ in $\operatorname{Sch}_{\mathbb{C}}$, the assignment $\left(f: W^{\prime} \rightarrow X\right) \mapsto$ $(f \circ \phi: W \rightarrow X)$ makes $\bar{E}$ a presheaf on $\mathbf{S c h}_{\mathbb{C}}$. One checks it defines a functor ext: $\operatorname{Funct}\left(\mathbf{S m}_{\mathbb{C}}^{\text {op }}, \mathcal{M}\right) \rightarrow \operatorname{Funct}\left(\mathbf{S c h}_{\mathbb{C}}^{\text {op }}, \mathcal{M}\right)$. In the opposite direction, we have rest: Funct $\left(\mathbf{S c h}_{\mathbb{C}}^{\text {op }}, \mathcal{M}\right) \rightarrow \operatorname{Funct}\left(\mathbf{S m}_{\mathbb{C}}^{\text {op }}, \mathcal{M}\right)$ and clearly rest $\circ$ ext $=\mathrm{Id}$. The transformation ext $\circ$ rest $\rightarrow$ Id is not an isomorphism in general.

Definition 5.1.2 Let $E$ be a presheaf on $\mathbf{S m}_{\mathbb{C}}$ of objects in Spc, Spc. or Spt. We define its semitopologization as the presheaf $\left.(\operatorname{ext}(E))^{\mathbf{s s t}}\right|_{\mathbf{S m}_{\mathbb{C}}}=\left.\bar{E}^{\mathbf{s s t}}\right|_{\mathbf{S m}}=$ rest $\circ \mathbf{s s t} \circ$ $\operatorname{ext}(E)$ on $\mathbf{S m}_{\mathbb{C}}$. The resulting presheaf is denoted by $E^{\text {sst }}$.

The semitopologization defines a natural transformation of functors $\operatorname{Id} \rightarrow(-)^{\text {sst }}$ on presheaves on $\mathbf{S m}_{\mathbb{C}}$. Immediately from Theorem 4.1.3, we get the following:

Proposition 5.1.3 Let $E \in \operatorname{Spt}(\mathbb{C})$. Then $E^{\text {sst }}$ is $\mathbb{A}^{1}$-weak-invariant.

Recall the following important tool from [11, Theorem 11], which is used in the form of Theorem 5.1.5.

Theorem 5.1.4 (Friedlander-Walker recognition principle) Let $E, F$ be presheaves of spectra on $\mathbf{S c h}_{\mathbb{C}}$ and let $f: E \rightarrow F$ be a morphism of presheaves, which is an objectwise weak-equivalence on $\mathbf{S m}_{\mathbb{C}}$. Then $\left|E\left(\Delta_{\top}^{\bullet}\right)\right| \rightarrow\left|F\left(\Delta_{\text {top }}^{\bullet}\right)\right|$ is a weak-equivalence.

Theorem 5.1.5 (1) If $f: E \rightarrow F$ is a morphism of presheaves of spectra on $\mathbf{S c h}_{\mathbb{C}}$, which is an objectwise weak-equivalence on $\mathbf{S m}_{\mathbb{C}}$, then $f^{\text {sst }}: E^{\text {sst }} \rightarrow F^{\text {sst }}$ is an objectwise weak-equivalence on $\mathbf{S m}_{\mathbb{C}}$.

(2) If $f: E \rightarrow F$ is a morphism of presheaves of spectra on $\mathbf{S m}_{\mathbb{C}}$, which is an objectwise weak-equivalence, then $f^{\text {sst }}: E^{\text {sst }} \rightarrow F^{\text {sst }}$ is an objectwise weakequivalence on $\mathbf{S m}_{\mathbb{C}}$. 
Proof We first prove (1). By the given assumption, for $X \in \mathbf{S m}_{\mathbb{C}}$, the map $E_{X} \rightarrow F_{X}$ is an objectwise weak-equivalence on $\mathbf{S m}_{\mathbb{C}}$. By Theorem 5.1.4, we have that the map $\left(E_{X}\right)^{\text {sst }}(\operatorname{Spec}(\mathbb{C})) \rightarrow\left(F_{X}\right)^{\text {sst }}(\operatorname{Spec}(\mathbb{C}))$ is a weak-equivalence. Now by Lemma 4.1.2, the map $E^{\text {sst }}(X) \rightarrow F^{\text {sst }}(X)$ is a weak-equivalence. To prove (2), note that the map $\bar{E} \rightarrow \bar{F}$ is an objectwise weak-equivalence on $\mathbf{S m}_{\mathbb{C}}$. So by (1) the map $\bar{E}^{\text {sst }}(X) \rightarrow$ $\bar{F}^{\text {sst }}(X)$ is a weak-equivalence for $X \in \mathbf{S m}_{\mathbb{C}}$. Equivalently, the map $E^{\text {sst }}(X) \rightarrow$ $F^{\text {sst }}(X)$ is a weak-equivalence.

\subsection{The loop space and the sst-functor}

For a map $f: E \rightarrow F$ of presheaves on $\mathbf{S m}_{\mathbb{C}}$ or $\mathbf{S c h}_{\mathbb{C}}$ of objects in $\mathbf{S p c}$, the fiber $\mathrm{fib}(f)$ is by definition $\lim \{\star \rightarrow F \leftarrow E\}$, and $\operatorname{fib}(f) \rightarrow E \rightarrow F$ is called a fiber sequence. This is not same as a homotopy fiber sequence unless $f$ is a fibration. Given an open or a closed immersion of schemes $A \subseteq B$ in $\mathbf{S m}_{\mathbb{C}}$, the functor $\Omega_{B / A}(-)$ on $\operatorname{Spt}(\mathbb{C})$ is $E \mapsto \Omega_{B / A} E=\left(\Omega_{B / A} E_{0}, \Omega_{B / A} E_{1}, \ldots\right)\left(\right.$ Section 3.3), where $\Omega_{B / A} E_{n}=$ $\mathcal{H o m}_{\bullet}\left(B / A, E_{n}\right)=\operatorname{fib}\left(\mathcal{H o m}\left(B, E_{n}\right) \rightarrow \mathcal{H o m}\left(A, E_{n}\right)\right)$. So we have an objectwise fiber sequence $\Omega_{B / A} E \rightarrow E_{B} \rightarrow E_{A}$ of presheaves, where $E_{B}(X)=E(B \times X)=$ $\mathcal{H} \operatorname{om}(B, E)(X)$. For $B \in \operatorname{Sm}_{\mathbb{C}}$, the map $\mathcal{H o m}(B, E) \rightarrow \mathcal{H o m}\left(B, E^{\text {sst }}\right)$ induces $\mathcal{H o m}(B, E)^{\mathrm{sst}} \rightarrow \mathcal{H o m}\left(B, E^{\mathrm{sst}}\right)$. By the universal property of $\Omega_{B / A}$, there is a natural transformation $\left(\Omega_{B / A}(-)\right)^{\text {sst }} \rightarrow \Omega_{B / A}\left((-)^{\text {sst }}\right)$.

Proposition 5.2.1 For $E \in \operatorname{Spt}(\mathbb{C})$, the map $\left(\Omega_{B / A} E\right)^{\mathrm{sst}} \rightarrow \Omega_{B / A}\left(E^{\mathrm{sst}}\right)$ in $\operatorname{Spt}(\mathbb{C})$ is an objectwise weak-equivalence on $\mathbf{S m}_{\mathbb{C}}$.

Proof For $E \in \operatorname{Spt}(\mathbb{C})$, let $\bar{E}=\operatorname{ext}(E)$, and $\bar{E}_{B}(X):=\bar{E}(B \times X)$ for $X \in \operatorname{Sch}_{\mathbb{C}}$. Let $\Omega_{B / A} \bar{E}$ be the objectwise fiber of $\bar{E}_{B} \rightarrow \bar{E}_{A}$. This sequence on $\mathbf{S c h}_{\mathbb{C}}$ restricts to $\Omega_{B / A} E \rightarrow E_{B} \rightarrow E_{A}$ on $\mathbf{S m}_{\mathbb{C}}$. Note that there is a morphism $\overline{E_{B}} \rightarrow \bar{E}_{B}$ of presheaves on $\mathbf{S c h}_{\mathbb{C}}$, which restricts to Id: $E_{B} \rightarrow E_{B}$ on $\mathbf{S m}_{\mathbb{C}}$. By the universal property of fiber, we get a morphism of presheaves $\Omega_{B / A}^{\prime} E:=\operatorname{fib}\left(\overline{E_{B}} \rightarrow \overline{E_{A}}\right) \rightarrow \Omega_{B / A} \bar{E}$ on $\mathbf{S c h}_{\mathbb{C}}$, which is an isomorphism on $\mathbf{S m}_{\mathbb{C}}$, which gives the commutative diagram of presheaves on $\mathbf{S c h}_{\mathbb{C}}$

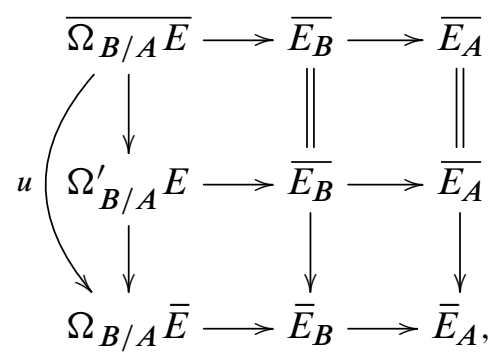


where the bottom two rows are objectwise fiber sequences and all vertical arrows are isomorphisms on $\mathbf{S m}_{\mathbb{C}}$. Let $u$ be the composition. Since filtered colimits commute with fiber products (see [26, Section IX.2 Theorem 1]), from the above we deduce the diagram

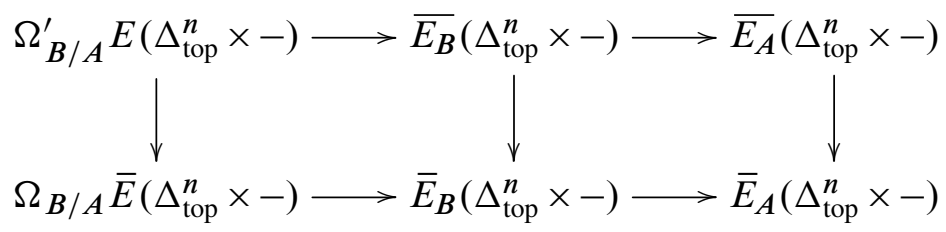

of presheaves of spectra on $\mathbf{S c h}_{\mathbb{C}}$, where the rows are objectwise fiber sequences. Since the fiber of a map of spectra is defined levelwise, taking the diagonals of maps of simplicial spectra as in Section 4.1.1, we get a commutative diagram

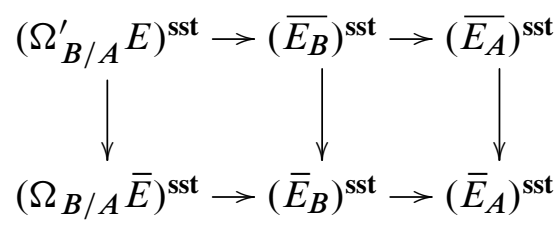

of presheaves of spectra on $\mathbf{S c h}_{\mathbb{C}}$, where the rows are objectwise fiber sequences. Since each vertical arrow in (5.2.1) is a morphism of presheaves of spectra on $\mathbf{S c h}_{\mathbb{C}}$ which is an isomorphism on $\mathbf{S m}_{\mathbb{C}}$, by Theorem 5.1.5 each vertical arrow in (5.2.2) is an objectwise weak-equivalence on $\mathbf{S m}_{\mathbb{C}}$. By definition and Lemma 4.1.2, the map $\left(\bar{E}_{B}\right)^{\text {sst }} \rightarrow\left(\bar{E}^{\text {sst }}\right)_{B}=\left(E^{\text {sst }}\right)_{B}$ is an isomorphism on $\mathbf{S m}_{\mathbb{C}}$, and the same is true for $E_{A}$. Composing these with the vertical maps in (5.2.2), and using the identification $\left(E_{B}\right)^{\text {sst }}=\left(\overline{E_{B}}\right)^{\text {sst }}$, we get a commutative diagram

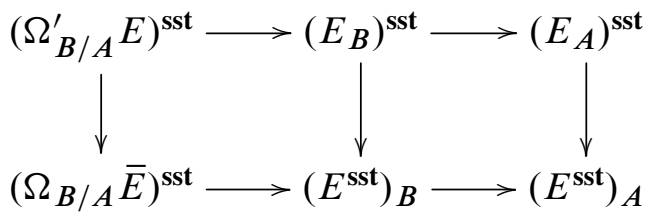

of presheaves of spectra on $\mathbf{S m}_{\mathbb{C}}$, where the rows are objectwise fiber sequences and the vertical arrows are objectwise weak-equivalences in $\mathbf{S m}_{\mathbb{C}}$. Consider the sequence of maps $\theta \circ u^{\text {sst }}:\left(\overline{\Omega_{B / A} E}\right)^{\text {sst }} \rightarrow\left(\Omega_{B / A} \bar{E}\right)^{\text {sst }} \rightarrow \Omega_{B / A}\left(\bar{E}^{\text {sst }}\right)$. The map $\theta$ is given by the universal property, and it is an isomorphism since the bottom row of (5.2.3) is an objectwise fiber sequence. The composite $\theta \circ u^{\text {sst }}$ is an objectwise weak-equivalence on $\mathbf{S m}_{\mathbb{C}}$ because the map $\overline{\Omega_{B / A} E} \rightarrow \Omega_{B / A} \bar{E}$ is an isomorphism on $\mathbf{S m}_{\mathbb{C}}$ as in (5.2.1) so Theorem 5.1.5 applies. Thus, by the two-out-of-three axiom, the map $u^{\text {sst }}$ 
is an objectwise weak-equivalence on $\operatorname{Sm}_{\mathbb{C}}$. Since $\left(\overline{\Omega_{B / A} E}\right)^{\text {sst }}=\left(\Omega_{B / A} E\right)^{\text {sst }}$ and $\Omega_{B / A}\left(\bar{E}^{\text {sst }}\right)=\Omega_{B / A}\left(E^{\text {sst }}\right)$, we are done.

Corollary 5.2.2 For $E \in \operatorname{Spt}(\mathbb{C})$, the maps $\left(\Omega_{t} E\right)^{\text {sst }} \rightarrow \Omega_{t}\left(E^{\text {sst }}\right)$ and $\left(\Omega_{T} E\right)^{\text {sst }} \rightarrow$ $\Omega_{T}\left(E^{\text {sst }}\right)$ in $\operatorname{Spt}(\mathbb{C})$ are objectwise weak-equivalences on $\mathbf{S m}_{\mathbb{C}}$.

The above follows by applying Proposition 5.2.1 to $t=\left(\mathbb{G}_{m}, 1\right)$ and $T=\left(\mathbb{P}^{1}, \infty\right)$. Using that $\Omega_{S^{1}} E(X)=\Omega_{S^{1}}(E(X))$ for a presheaf of spectra $E$ on $\mathbf{S c h}_{\mathbb{C}}$ and that $\Omega_{S^{1}} \bar{F} \cong \overline{\Omega_{S^{1}} F}$ for a presheaf of spectra $F$ on $\mathbf{S m}_{\mathbb{C}}$, one checks that for a presheaf $E$ of spectra on $\mathbf{S m}_{\mathbb{C}}$, the map $\left(\Omega_{S^{1}} E\right)^{\text {sst }} \rightarrow \Omega_{S^{1}}\left(E^{\text {sst }}\right)$ is an isomorphism.

\section{Homotopy semitopologization}

We prove that the classes of $\mathrm{BG}$ and $\mathbb{A}^{1}-\mathrm{BG}$ presheaves of spectra are closed under semitopologization. We prove similar results for $(s, \mathfrak{p})$-bispectra. Using these we define homotopy semitopologization on motivic homotopy categories.

\subsection{On $\mathcal{S H}_{S^{1}}(\mathbb{C})$}

For a simplicial spectrum $E$, let $E_{p}:=E(\Delta[p])$ for $p \geq 0$.

Lemma 6.1.1 If each $E_{p}$ of a simplicial spectrum $E$ is cofibrant, then so is $\operatorname{diag} E$.

Proof By Theorem 2.2.1, we need to show each map $S^{1} \wedge(\operatorname{diag} E)_{n} \rightarrow(\operatorname{diag} E)_{n+1}$ in Spc . is a monomorphism. For a monomorphism $A \rightarrow B$ in Spc, one has $(B / A)_{n}=$ $B_{n} / A_{n}$. For $A, B \in \mathbf{S p c}_{\bullet}$, one has $(A \times B)_{n}=A_{n} \times B_{n}$ and $(A \wedge B)_{n}=A_{n} \wedge B_{n}$. So it suffices to show $\left(S^{1}\right)_{p} \wedge(\operatorname{diag} E)_{n, p} \rightarrow(\operatorname{diag} E)_{n+1, p}$ is a monomorphism. But $(\operatorname{diag} E)_{n, p}=E_{p, p}^{n}(\operatorname{Section} 4.1 .1)$ and $\left(S^{1}\right)_{i} \wedge E_{p, i}^{n} \rightarrow E_{p, i}^{n+1}$ is a monomorphism because each $E_{p}$ is cofibrant. Thus, the assertion follows.

Lemma 6.1.2 If each $f_{p}: E_{p} \rightarrow F_{p}$ of a morphism $f: E \rightarrow F$ of simplicial spectra is a cofibration of spectra, then the map $\operatorname{diag} E \rightarrow \operatorname{diag} F$ ie $|E| \rightarrow|F|$ is a cofibration.

Proof A cofibration of spectra is also a levelwise monomorphism in Spc $\mathbf{S}_{\bullet}$. So $f$ is a levelwise monomorphism of bisimplicial sets, and the map $\operatorname{diag} E \rightarrow \operatorname{diag} F$ is a levelwise monomorphism in Spc. By Section 2.2.1 and Theorem 2.2.1, we need to show that the spectrum $\operatorname{diag} F / \operatorname{diag} E$ is cofibrant, where $(\operatorname{diag} F / \operatorname{diag} E)_{n}=$ $(\operatorname{diag} F)_{n} /(\operatorname{diag} E)_{n}$. Let $G=F / E$, where $G_{p, q}^{n}=F_{p, q}^{n} / E_{p . q}^{n}$. Since

$$
\left(S^{1} \wedge F_{p}^{n}\right) /\left(S^{1} \wedge E_{p}^{n}\right) \simeq S^{1} \wedge\left(F_{p}^{n} / E_{p}^{n}\right),
$$


we see that $G$ is a simplicial spectrum. Furthermore,

$(\operatorname{diag} G)_{n, p}=G_{p, p}^{n}=F_{p, p}^{n} / E_{p, p}^{n}=(\operatorname{diag} F)_{n, p} /(\operatorname{diag} E)_{n, p}=(\operatorname{diag} F / \operatorname{diag} E)_{n, p}$.

Hence $\operatorname{diag} G=\operatorname{diag} F / \operatorname{diag} E$. Hence by Lemma 6.1.1, it suffices to show that $G_{p}$ is a cofibrant spectrum. But, $G_{p}=F_{p} / E_{p}$, and that $E_{p} \rightarrow F_{p}$ is a cofibration implies that $F_{p} / E_{p}$ is cofibrant.

Since Nisnevich or motivic cofibrations between presheaves of spectra on $\mathbf{S m}_{S}$ are exactly objectwise cofibrations, we deduce the following from Lemma 6.1.2.

Corollary 6.1.3 If each $f_{p}: E_{p} \rightarrow F_{p}$ of a morphism $f: E \rightarrow F$ of presheaves of simplicial spectra on $\mathbf{S c h}_{\mathbb{C}}$ is an objectwise (Nisnevich, motivic) cofibration of presheaves of spectra, then the map $\operatorname{diag} E \rightarrow \operatorname{diag} F$ ie $|E| \rightarrow|F|$ is an objectwise (Nisnevich, motivic) cofibration.

Proposition 6.1.4 Let $g \circ f: E \rightarrow F \rightarrow G$ be an objectwise homotopy cofiber sequence of presheaves of spectra on $\mathbf{S c h}_{\mathbb{C}}$. Then $g^{\text {sst }} \circ f^{\text {sst }}: E^{\text {sst }} \rightarrow F^{\text {sst }} \rightarrow G^{\text {sst }}$ is an objectwise homotopy cofiber sequence on $\mathbf{S m}_{\mathbb{C}}$.

Proof Recall from [2, Section A2] that $g \circ f: E \rightarrow F \rightarrow G$ is an objectwise homotopy cofiber sequence if and only if we have a sequence $g^{\prime} \circ f^{\prime}: E \rightarrow H \rightarrow H / E$ of presheaves of spectra on $\mathbf{S c h}_{\mathbb{C}}, h: H \rightarrow F, p: H / E \rightarrow G$, where $f^{\prime}$ is an objectwise cofibration, $h, p$ are objectwise weak-equivalences, such that $h \circ f^{\prime}=f$ and $p \circ g^{\prime}=$ $g \circ h$. By Theorem 5.1.5, $h^{\text {sst }}$ and $p^{\text {sst }}$ are objectwise weak-equivalences on $\mathbf{S m}_{\mathbb{C}}$. Using Proposition 4.2.1(3), it remains to show that the map $f^{\text {sst }}: E^{\text {sst }} \rightarrow H^{\text {sst }}$ is an objectwise cofibration, equivalently, that the $\operatorname{map} \operatorname{diag}(\widetilde{E}) \rightarrow \operatorname{diag}(\tilde{H})$ is a cofibration, where $\widetilde{E}$ is the presheaf of simplicial spectra on $\operatorname{Sch}_{\mathbb{C}}$ defined by $\widetilde{E}(\Delta[p])(-)=$ $E\left(\Delta_{\text {top }}^{p} \times-\right)$ (Definition 4.1.1) and similarly for $\tilde{H}$. Since $\tilde{E}(\Delta[p]) \rightarrow \widetilde{H}(\Delta[p])$ is a filtered colimit of objectwise cofibrations, by Mitchell [29, Proposition 3.2] this map is an objectwise cofibration. Hence, by Corollary 6.1 .3 , the map $\operatorname{diag}(\widetilde{E}) \rightarrow \operatorname{diag}(\tilde{H})$ is an objectwise cofibration. This finishes the proof.

Theorem 6.1.5 Let $E$ be a presheaf of spectra (or complexes of abelian groups) on $\mathbf{S m}_{\mathbb{C}}$. If $E$ is $B G$, then so is $E^{\mathrm{sst}}$. If $E$ is $\mathbb{A}^{1}-B G$, then so is $E^{\mathbf{s s t}}$.

Proof We prove it for presheaves of spectra since the other is a special case via Dold-Kan correspondence. We prove the first statement. Via the artificial extension in Definition 5.1.1, regard $E$ as a presheaf on $\mathbf{S c h}_{\mathbb{C}}$. Given $X \in \mathbf{S m}_{\mathbb{C}}$, we have that the presheaf $E_{X}$ on $\operatorname{Sch}_{\mathbb{C}}$ is $E_{X}(Y):=E(X \times Y)$ for $Y \in \mathbf{S c h}_{\mathbb{C}}$. Given a 
Nisnevich square as in (3.0.1), where $X, U, V, W \in \mathbf{S m}_{\mathbb{C}}$ with $W=U \times{ }_{X} V$, we have a commutative diagram

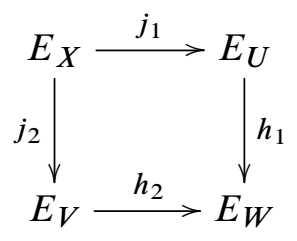

of presheaves of spectra on $\mathbf{S c h}_{\mathbb{C}}$, which is objectwise homotopy Cartesian on $\mathbf{S m}_{\mathbb{C}}$. Equivalently, it is objectwise homotopy co-Cartesian on $\mathbf{S m}_{\mathbb{C}}$. Let $G_{1}$ and $G_{2}$ be the objectwise homotopy cofibers of $j_{1}$ and $h_{2}$. Then (6.1.1) is objectwise homotopy co-Cartesian on $\mathbf{S m}_{\mathbb{C}}$ if and only if the map $h: G_{1} \rightarrow G_{2}$ is an objectwise weakequivalence on $\mathbf{S m}_{\mathbb{C}}$. So, by Theorem 5.1.5, we have that the map $h^{\text {sst }}: G_{1}^{\text {sst }} \rightarrow G_{2}^{\text {sst }}$ is an objectwise weak-equivalence on $\mathbf{S m}_{\mathbb{C}}$. Using Proposition 6.1.4, we obtain a commutative diagram

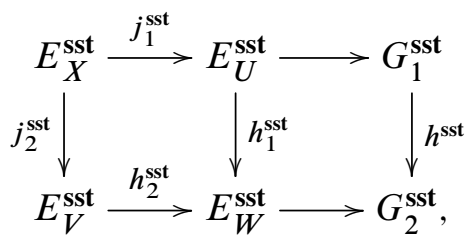

where the rows are objectwise homotopy cofiber sequences of presheaves on $\mathbf{S m}_{\mathbb{C}}$. Since $h^{\text {sst }}$ is an objectwise weak-equivalence on $\mathbf{S m}_{\mathbb{C}}$, the left square in (6.1.2) is objectwise homotopy co-Cartesian on $\mathbf{S m}_{\mathbb{C}}$. Equivalently, it is objectwise homotopy Cartesian on $\mathbf{S m}_{\mathbb{C}}$. Evaluating at $\operatorname{Spec}(\mathbb{C})$ and applying Lemma 4.1.2, we obtain

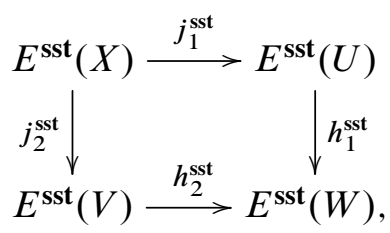

a homotopy Cartesian square of spectra. This shows that $E^{\text {sst }}$ is BG as desired. The second statement follows from the first and Proposition 5.1.3.

Applying Theorems 3.1.5, 5.1.5 and 6.1.5, we conclude:

Corollary 6.1.6 The sst of an $S^{1}$-stable motivic weak-equivalence of $\mathbb{A}^{1}-B G$ motivic spectra is an objectwise weak-equivalence of $\mathbb{A}^{1}-B G$ motivic spectra.

Corollary 6.1.7 There exists an endofunctor host: $\mathcal{S H}_{S^{1}}(\mathbb{C}) \rightarrow \mathcal{S H}_{S^{1}}(\mathbb{C})$, which coincides with the sst-functor on $\mathbb{A}^{1}-B G$ motivic spectra up to isomorphism. 
Proof We know from Theorem 6.1.5 that sst: $\operatorname{Spt}(\mathbb{C}) \rightarrow \operatorname{Spt}(\mathbb{C})$ preserves $\mathbb{A}^{1}-\mathrm{BG}$ motivic spectra. Since an $S^{1}$-stable motivic fibrant motivic spectrum is Nisnevich fibrant and $\mathbb{A}^{1}$-local, it is $\mathbb{A}^{1}-\mathrm{BG}$ by Lemma 3.1.3. By Corollary 6.1.6, we know sst takes a trivial motivic fibration between $S^{1}$-stable motivic fibrant motivic spectra into an $S^{1}$-stable motivic weak-equivalence. Thus, by [14, Proposition 8.4.8] we obtain a right derived endofunctor host: $\mathcal{S H}_{S^{1}}(\mathbb{C}) \rightarrow \mathcal{S H}_{S^{1}}(\mathbb{C})$, with desired properties.

\subsection{On $\mathcal{S H}(\mathbb{C})$}

Let $E=\left(E_{0}, E_{1}, \ldots\right) \in \mathbf{S p t}_{(s, \mathfrak{p})}(\mathbb{C})$ (Sections 2.3.1 and 2.3.2) with the bonding maps $T \wedge E_{n} \rightarrow E_{n+1}$. It yields $\left(T \wedge E_{n}\right)^{\text {sst }}=T^{\text {sst }} \wedge E_{n}^{\text {sst }} \rightarrow E_{n+1}^{\text {sst }}$, by Proposition 4.2.1. Composed with $T \wedge E_{n}^{\text {sst }} \rightarrow T^{\text {sst }} \wedge E_{n}^{\text {sst }}$, we get $T \wedge E_{n}^{\text {sst }} \rightarrow E_{n+1}^{\text {sst }}$. This gives $E^{\text {sst }}:=$ $\left(E_{0}^{\mathrm{sst}}, E_{1}^{\mathrm{sst}}, \ldots\right) \in \mathbf{S p t}_{(s, \mathfrak{p})}(\mathbb{C})$. One checks $\mathrm{Id} \rightarrow(-)^{\text {sst }}$ is natural on $\mathbf{S p t}_{(s, \mathfrak{p})}(\mathbb{C})$.

Theorem 6.2.1 (1) The class of $\mathbb{A}^{1}-B G(s, \mathfrak{p})$-bispectra is closed under the sstfunctor.

(2) The class of $\mathbb{A}^{1}-B G$ motivic $\Omega_{T}$-bispectra (Definition 3.3.2) is closed under the sst-functor.

(3) If $f$ is a stable motivic weak-equivalence of $\mathbb{A}^{1}-B G$ motivic $\Omega_{T}$-bispectra, then $f^{\text {sst }}$ is a $T$-levelwise objectwise weak-equivalence of $\mathbb{A}^{1}-B G$ motivic $\Omega_{T}$-bispectra.

Proof Part (1) holds by Theorem 6.1.5. For (2), let $E$ be an $\mathbb{A}^{1}-\mathrm{BG}$ motivic $\Omega_{T^{-}}$ bispectrum. Using Lemma 3.3.1 we deduce that each $\Omega_{T} E_{n}$ is an $\mathbb{A}^{1}-\mathrm{BG}$ motivic $S^{1}$-spectrum. So by Theorem 3.1.5 and Corollary 6.1.6, the map $E_{n}^{\text {sst }} \rightarrow\left(\Omega_{T} E_{n+1}\right)^{\text {sst }}$ is an objectwise weak-equivalence. Now by Corollary 5.2.2, the map $\left(E^{\text {sst }}\right)_{n} \rightarrow$ $\Omega_{T}\left(\left(E^{\text {sst }}\right)_{n+1}\right)$ is an objectwise weak-equivalence, thus an $S^{1}$-stable motivic weakequivalence. Part (3) follows from (1), (2) and Theorems 3.3.3 and 5.1.5.

Recall that for a morphism $f: E \rightarrow F$ in $\operatorname{Spt}_{(s, \mathfrak{p})}(\mathbb{C})$, the cone $C(f)$ is the pushout

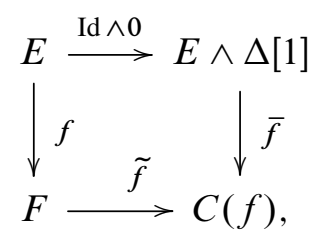

where $\Delta[1]$ is pointed by one. Collapsing $F$ to the base point of $\Sigma_{S} E=E \wedge S^{1}$ and using the quotient map $E \wedge \Delta[1] \rightarrow E \wedge S^{1}$, we get $\delta_{f}: C(f) \rightarrow \Sigma_{s} E$, which gives $\delta_{f} \circ \widetilde{f} \circ f: E \rightarrow F \rightarrow C(f) \rightarrow \Sigma_{s} E$. 
Lemma 6.2.2 Let $f: E \rightarrow F$ be a morphism in $\mathbf{S p t}_{(s, \mathfrak{p})}(\mathbb{C})$. Then the following is a pushout square:

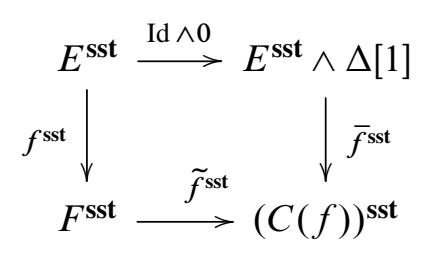

Proof For a presheaf $G$ on $\mathbf{S m}_{\mathbb{C}}$ of objects in $\mathbf{S p c}_{\bullet}$ or Spt. Let $\bar{G}$ be its artificial extension on $\mathbf{S c h}_{\mathbb{C}}$ as in Definition 5.1.1. This extends $(s, \mathfrak{p})$-bispectra over $\mathbf{S m}_{\mathbb{C}}$ to $(s, \mathfrak{p})$-bispectra over $\mathbf{S c h}_{\mathbb{C}}$. Note the pushout of a diagram of presheaves of $(s, \mathfrak{p})-$ bispectra is defined objectwise, and one has $\overline{E \wedge \Delta[1]} \simeq \bar{E} \wedge \Delta[1]$. Since the artificial extension is defined as a colimit and since the colimits commute among themselves (cf [26, Section IX.8]), the pushout (6.2.1) (a colimit) remains a pushout square if we replace the presheaves by their artificial extensions. So we may assume the presheaves $E$ and $F$ are defined on $\mathbf{S c h}_{\mathbb{C}}$. The commutativity of two colimits also implies that the diagram

$$
E\left(\Delta_{\text {top }}^{n} \times-\right) \stackrel{\text { Id } \wedge 0}{\longrightarrow} E\left(\Delta_{\text {top }}^{n} \times-\right) \wedge \Delta[1]
$$

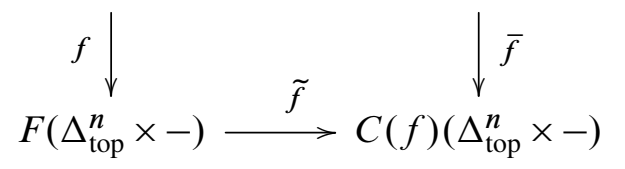

is a pushout square. Since $\operatorname{Hom}_{\mathcal{C}}\left(X \wedge \Delta[k]_{+}, Y\right) \simeq \operatorname{Hom}_{\mathcal{C}}\left(X, \mathcal{H o m} \bullet\left(\Delta[k]_{+}, Y\right)\right)$ where $\mathcal{C}$ is the category of $(s, \mathfrak{p})$-bispectra on $\mathbf{S c h}_{\mathbb{C}}$, we deduce that (6.2.3) remains a pushout square after smashing with $\Delta[k]_{+}$for $k \geq 0$. Since a coequalizer (a colimit) commutes with colimits, by Section 4.1.1 we obtain a pushout square (6.2.2) except $E^{\text {sst }} \wedge \Delta[1]$ is replaced with $(E \wedge \Delta[1])^{\text {sst }}$. But, by Proposition 4.2.1(4) and the isomorphism $\Delta[1] \simeq(\Delta[1])^{\text {sst }}$, we do have $E^{\text {sst }} \wedge \Delta[1] \simeq(E \wedge \Delta[1])^{\text {sst }}$.

Theorem 6.2.3 There exists a triangulated endofunctor host: $\mathcal{S H}(\mathbb{C}) \rightarrow \mathcal{S H}(\mathbb{C})$, which coincides with the sst-functor on $\mathbb{A}^{1}-B G$ motivic $\Omega_{T}$-bispectra up to isomorphism.

Proof By Theorem 6.2.1, we know $\mathbb{A}^{1}$-BG motivic $\Omega_{T}$-bispectra are closed under sst. By [30, Lemma 2.3.8], the functor $\Sigma_{T}: \operatorname{Spt}(\mathbb{C}) \rightarrow \operatorname{Spt}(\mathbb{C})$ preserves stable motivic weak-equivalences and cofibrations. Hence, $\Sigma_{T}$ is a left Quillen endofunctor with the right adjoint $\Omega_{T}: \operatorname{Spt}(\mathbb{C}) \rightarrow \operatorname{Spt}(\mathbb{C})$. An $(s, \mathfrak{p})$-bispectrum $E=\left(E_{0}, E_{1}, \ldots\right)$ is 
stable motivic fibrant if and only if it is a motivic $\Omega_{T}$-bispectrum and it is $T$-levelwise $S^{1}$-stable motivic fibrant (cf [15, Definition 3.1, Theorem 3.4]). So a stable motivic fibrant $(s, \mathfrak{p})$-bispectrum is an $\mathbb{A}^{1}-\mathrm{BG}$ motivic $\Omega_{T}$-bispectrum. By Theorem 6.2.1, we know sst takes a trivial stable motivic fibration between stable motivic fibrant $(s, \mathfrak{p})-$ bispectra to a stable motivic weak-equivalence. Thus, by [14, Proposition 8.4.8] we obtain a right derived endofunctor host: $\mathcal{S H}(\mathbb{C}) \rightarrow \mathcal{S H}(\mathbb{C})$ with desired properties. We now check that host: $\mathcal{S H}(\mathbb{C}) \rightarrow \mathcal{S H}(\mathbb{C})$ is a triangulated functor. Since host preserves finite coproducts and products in $\mathcal{S H}(\mathbb{C})$, it is an additive functor. The shift $E \mapsto E[1]$ on $\mathcal{S H}(\mathbb{C})$ is given by the functor $E \mapsto \Sigma_{s} E$. One sees that host commutes with $\Sigma_{S}$ by Proposition 4.2.1 and the isomorphism $S^{1} \simeq\left(S^{1}\right)^{\text {sst }}$. For a distinguished triangle in $\mathcal{S H}(\mathbb{C})$ of the form $E \rightarrow F \rightarrow C(f) \rightarrow \Sigma_{s} E$ for a map $f: E \rightarrow F$ in $\mathbf{S p t}_{(s, \mathfrak{p})}(\mathbb{C})$ (cf Østvær, Röndigs and Voevodsky [34, Section 2.3]), by Lemma 6.2.2 and the isomorphism $\left(\Sigma_{s} E\right)^{\text {sst }} \simeq \Sigma_{s} E^{\text {sst }}$, we deduce that $E^{\text {sst }} \rightarrow F^{\text {sst }} \rightarrow(C(f))^{\text {sst }} \rightarrow \Sigma_{s} E^{\text {sst }}$ is also a distinguished triangle in $\mathcal{S H}(\mathbb{C})$.

Definition 6.2.4 For the rest of this paper, we call the functor host of Corollary 6.1.7 and Theorem 6.2 .3 by the name homotopy semitopologization functor. For any $E$ in $\mathcal{S H} \mathcal{S}^{1}(\mathbb{C})$ or $\mathcal{S H}(\mathbb{C})$, we denote $\operatorname{host}(E)$ by $E^{\text {host }}$.

\section{Representing semitopological $K$-theory in $\mathcal{S H}(\mathbb{C})$}

We prove that the semitopological $K$-theory of [8] is representable in motivic homotopy categories. For $\mathcal{S H}_{S^{1}}(\mathbb{C})$, it is easy by semitopologizing an $\mathbb{A}^{1}-$ BG presheaf of spectra representing the algebraic $K$-theory. For $\mathcal{S H}(\mathbb{C})$, an essential thing is to find an $\mathbb{A}^{1}-\mathrm{BG}$ motivic $\Omega_{T}$-bispectrum that represents algebraic $K$-theory; see Proposition 7.2.3.

The semitopological $K$-theory of a complex variety $X$ is a bridge between the algebraic and the topological $K$-theories of $X$. This theory was defined in [9] as the stable homotopy groups of an infinite loop space, constructed out of the stabilization of the analytic space of algebraic morphisms of complex varieties. In [8], another definition of the semitopological $K$-theory is given by $K_{p}^{\text {sst }}(X):=\pi_{p}\left(\left|\mathcal{K}\left(\Delta_{\text {top }}^{\bullet} \times X\right)\right|\right)$ for $p \in \mathbb{Z}$, where $\mathcal{K}(-)$ is the presheaf of connective spectra on $\mathbf{S c h}_{\mathbb{C}}$ that represents Quillen algebraic $K$-theory. By [8, Theorem 1.4], this definition coincides with the original one in [9] for projective weakly normal varieties.

\subsection{Representability in $\mathcal{S H}_{S^{1}}(\mathbb{C})$}

Recall that Jardine [19] (see also Kim [20]) constructed a presheaf of spectra on $\mathbf{S m}_{\mathbb{C}}$ that represents the algebraic $K$-theory. This construction and some properties 
are summarized as follows, taken from Jardine [19, Theorem 5, Proposition 9], and Thomason and Trobaugh [40, Proposition 6.8, Theorem 10.8]:

Theorem 7.1.1 There is a presheaf $\mathcal{K}$ of spectra on $\mathbf{S m}_{\mathbb{C}}$ such that for $X \in \mathbf{S m}_{\mathbb{C}}$, $\mathcal{K}(X)$ represents the algebraic $K$-theory of $X$. This is a presheaf of $\Omega_{s}-$ spectra above level zero, equipped with smash product $\mathcal{K}_{i} \wedge \mathcal{K}_{j} \rightarrow \mathcal{K}_{i+j}$ which commutes with the bonding maps of $\mathcal{K}$. Furthermore, $\mathcal{K}$ is an $\mathbb{A}^{1}-B G$ presheaf of spectra on $\mathbf{S m}_{\mathbb{C}}$.

For representability of semitopological $K$-theory in $\mathcal{S H}_{S^{1}}(\mathbb{C})$ (and $\mathcal{H}_{\bullet}(\mathbb{C})$ ), we have a quick answer. Let $\mathcal{K}$ be presheaf of spectra on $\mathbf{S m}_{\mathbb{C}}$ as in Theorem 7.1.1.

Proposition 7.1.2 Let $X \in \mathbf{S m}_{\mathbb{C}}$ and $p \in \mathbb{Z}$. Then $K_{p}^{\text {sst }}(X) \simeq\left[\Sigma_{S}^{\infty} X_{+}[p], \mathcal{K}^{\text {sst }}\right]_{\mathbb{A}^{1}}$. That is, the semitopological $K$-theory is representable in $\mathcal{S H}_{S^{1}}(\mathbb{C})$.

Proof It holds by Corollary 3.1.6, Theorems 6.1.5, 7.1.1 and the definition of $K_{p}^{\text {sst }}$.

Corollary 7.1.3 For $X \in \mathbf{S m}_{\mathbb{C}}$ and $p \geq 0$, we have $K_{p}^{\text {sst }}(X) \simeq\left[\Sigma_{s}^{p} X_{+}, \boldsymbol{R} \operatorname{Ev}_{0} \mathcal{K}^{\text {sst }}\right]_{\mathbb{A}^{1}}$. That is, the semitopological $K$-theory is representable in $\mathcal{H}_{\bullet}(\mathbb{C})$.

\subsection{Representability in $\mathcal{S H}(\mathbb{C})$}

For a presheaf of spectra $E=\left(E_{0}, E_{1}, \ldots\right)$ on $\mathbf{S m}_{\mathbb{C}}$, let $E\{n\}$ be the presheaf of spectra $\left(E_{n}, E_{n+1}, \ldots\right)$. We use $\mathcal{K}$ of Section 7.1 in what follows. Let $f: \mathcal{K} \rightarrow \mathcal{K}^{\mathrm{fib}} \leftarrow$ $\mathcal{K}^{\mathrm{cf}}: g$ be two morphisms in $\operatorname{Spt}(\mathbb{C})$, where $f$ is an $S^{1}$-stable motivic fibrant replacement of $\mathcal{K}$ and $g$ is an $S^{1}$-stable motivic cofibrant replacement of $\mathcal{K}^{\text {fib }}$. Since $\mathcal{K}^{\text {fib }}$ is motivic fibrant and $g$ is a motivic fibration, it follows that $\mathcal{K}^{\mathrm{cf}}$ is motivic cofibrantfibrant. Moreover, by Theorem 2.2.1 and Corollary 3.1.8, each $\mathcal{K}^{\mathrm{cf}}\{n\}$ is motivic cofibrant-fibrant. By Theorem 7.1.1 and Corollary 3.1.8, the maps $\mathcal{K}_{n} \rightarrow \mathcal{K}_{n}^{\text {fib }} \leftarrow \mathcal{K}_{n}^{\text {cf }}$ are objectwise weak-equivalences for each $n \geq 1$. Using the product structure on $\mathcal{K}$ in Theorem 7.1.1, we obtain a morphism of motivic spectra $\mathcal{K}^{\mathrm{cf}} \wedge \mathcal{K}_{1}^{\mathrm{cf}} \rightarrow \mathcal{K}^{\mathrm{cf}}\{1\}$ in $\mathcal{S H} \mathcal{H}^{1}(\mathbb{C})$. This is equivalent to a morphism $\mathcal{K}^{\mathrm{cf}} \rightarrow \boldsymbol{R} \Omega_{\mathcal{K}_{1}^{\mathrm{cf}}} \mathcal{K}^{\mathrm{cf}}\{1\} \simeq \Omega_{\mathcal{K}_{1}^{\mathrm{cf}}} \mathcal{K}^{\mathrm{cf}}\{1\}$ in $\mathcal{S} \mathcal{H}_{S^{1}}(\mathbb{C})$. Since $\mathcal{K}^{\mathrm{cf}}$ is cofibrant and $\Omega_{\mathcal{K}_{1}^{\mathrm{cf}}} \mathcal{K}^{\mathrm{cf}}\{1\}$ is fibrant, this map lifts to a map in $\operatorname{Spt}(\mathbb{C})$. Taking the adjoint of this map, we conclude that there is a morphism $\phi: \mathcal{K}^{\mathrm{cf}} \wedge \mathcal{K}_{1}^{\mathrm{cf}} \rightarrow \mathcal{K}^{\mathrm{cf}}\{1\}$ in $\mathbf{S p t}(\mathbb{C})$. Thus, we obtained a cofibrant-fibrant motivic spectrum model $\mathcal{K}^{\text {cf }}$ for the algebraic $K$-theory, with a product that yields a ring structure on $K_{*}(X)$ for $X \in \mathbf{S m}_{\mathbb{C}}$. The above product structure on the presheaf $\mathcal{K}^{\text {cf }}$ of spectra allows one to construct a $T$-spectrum that represents the algebraic $K$ theory in $\mathcal{S H}(\mathbb{C})$. For details, we refer to [20]. To prove the representability of the semitopological $K$-theory in $\mathcal{S H}(\mathbb{C})$, we lift this $T$-spectrum to an $(s, \mathfrak{p})$-bispectrum, for which we recycle the construction in [41, Section 6.2]. 


\section{Lemma 7.2.1 Let $X \in \mathbf{S m}_{\mathbb{C}}$.}

(1) For $p \geq m \geq 0$, we have $\left[\Sigma_{s}^{p} X_{+}, \mathcal{K}_{m}^{\mathrm{cf}}\right]_{\mathbb{A}^{1}} \simeq K_{p-m}(X)$ and a split exact sequence

$$
0 \rightarrow\left[\Sigma_{s}^{p} \Sigma_{T} X_{+}, \mathcal{K}_{m}^{\mathrm{cf}}\right]_{\mathbb{A}^{1}} \rightarrow K_{p-m}\left(\mathbb{P}_{X}^{1}\right) \rightarrow K_{p-m}(X) \rightarrow 0 .
$$

(2) For $0 \leq p<m,\left[\Sigma_{s}^{p} X_{+}, \mathcal{K}_{m}^{\mathrm{cf}}\right]_{\mathbb{A}^{1}}=\left[\Sigma_{s}^{p} \Sigma_{T} X_{+}, \mathcal{K}_{m}^{\mathrm{cf}}\right]_{\mathbb{A}^{1}}=0$ and there is a split exact sequence

$$
0 \rightarrow\left[\Sigma_{s}^{p} \Sigma_{T} X_{+}, \mathcal{K}_{m}^{\mathrm{cf}}\right]_{\mathbb{A}^{1}} \rightarrow K_{p-m}\left(\mathbb{P}_{X}^{1}\right) \rightarrow K_{p-m}(X) \rightarrow 0 .
$$

Proof For $p \geq 0$, the cofiber sequence $\Sigma_{s}^{\infty} \Sigma_{s}^{p} X_{+} \rightarrow \Sigma_{S}^{\infty} \Sigma_{S}^{p}\left(\mathbb{P}_{X}^{1}\right)_{+} \rightarrow \Sigma_{S}^{\infty} \Sigma_{s}^{p} \Sigma_{T} X_{+}$ in $\mathcal{S H}_{S^{1}}(\mathbb{C})$ (cf [34, Lemma 2.16]) and Lemma 3.1.2 give us a long exact sequence

$$
\rightarrow\left[\Sigma_{s}^{\infty} \Sigma_{s}^{p} \Sigma_{T} X_{+}, \mathcal{K}^{\mathrm{cf}}\right]_{\mathbb{A}^{1}} \rightarrow K_{p}\left(\mathbb{P}_{X}^{1}\right) \rightarrow K_{p}(X) \rightarrow,
$$

where the map $i_{0}^{*}: K_{p}\left(\mathbb{P}_{X}^{1}\right) \rightarrow K_{p}(X)$ splits by the pullback via the projection $X \times$ $\mathbb{P}^{1} \rightarrow X$. Part (1) follows easily from this and the adjoint isomorphisms

$$
\left[\Sigma_{s}^{\infty} A, \mathcal{K}^{\mathrm{cf}}\right]_{\mathbb{A}^{1}} \simeq\left[A, \mathcal{K}_{0}^{\mathrm{cf}}\right]_{\mathbb{A}^{1}} \simeq\left[A, \Omega_{s}^{m} \mathcal{K}_{m}^{\mathrm{cf}}\right]_{\mathbb{A}^{1}} \simeq\left[\Sigma_{s}^{m} A, \mathcal{K}_{m}^{\mathrm{cf}}\right]_{\mathbb{A}^{1}}
$$

for $A \in \mathbf{S p c}_{\bullet}(\mathbb{C})$. Notice here that $\mathcal{K}^{\mathrm{cf}}$ and $\mathcal{K}_{m}^{\mathrm{cf}}$ are all motivic (hence objectwise) fibrant and $\mathcal{K}^{\mathrm{cf}}$ is a motivic $\Omega_{s}$-spectrum. To prove the first part of (2), first use Lemma 3.1.2 and Corollary 3.1.8 to obtain isomorphisms

$$
\left[\Sigma_{s}^{p} X_{+}, \mathcal{K}_{m}^{\mathrm{cf}}\right]_{\mathbb{A}^{1}} \simeq \pi_{p}\left(\mathcal{K}_{m}^{\mathrm{cf}}(X)\right) \simeq \pi_{p-m}\left(\mathcal{K}^{\mathrm{cf}}(X)\right),
$$

where the last term is zero if $p-m<0$ since $\mathcal{K}^{\mathrm{cf}}(X)$ is a connective spectrum. For the second part of (2), from the cofiber sequence $\Sigma_{S}^{\infty} \Sigma_{S}^{p} X_{+} \rightarrow \Sigma_{S}^{\infty} \Sigma_{S}^{p}\left(\mathbb{P}_{X}^{1}\right)_{+} \rightarrow$ $\Sigma_{S}^{\infty} \Sigma_{s}^{p} \Sigma_{T} X_{+}$, we get an exact sequence

$$
\begin{aligned}
{\left[\Sigma_{s}^{\infty} \Sigma_{s}^{p+1}\left(\mathbb{P}_{X}^{1}\right)_{+}, \mathcal{K}^{\mathrm{cf}}\{m\}\right]_{\mathbb{A}^{1}} \rightarrow\left[\Sigma_{s}^{\infty} \Sigma_{s}^{p+1} X_{+}, \mathcal{K}^{\mathrm{cf}}\{m\}\right]_{\mathbb{A}^{1}} } \\
\rightarrow\left[\Sigma_{s}^{\infty} \Sigma_{s}^{p} \Sigma_{T} X_{+}, \mathcal{K}^{\mathrm{cf}}\{m\}\right]_{\mathbb{A}^{1}} \rightarrow\left[\Sigma_{s}^{\infty} \Sigma_{s}^{p}\left(\mathbb{P}_{X}^{1}\right)_{+}, \mathcal{K}^{\mathrm{cf}}\{m\}\right]_{\mathbb{A}^{1}} .
\end{aligned}
$$

By Corollary 3.1.8 and the adjointness, this exact sequence is equivalent to

$$
\begin{aligned}
{\left[\Sigma_{s}^{p+1}\left(\mathbb{P}_{X}^{1}\right)_{+}, \mathcal{K}_{m}^{\mathrm{cf}}\right]_{\mathbb{A}^{1}} \rightarrow\left[\Sigma_{s}^{p+1} X_{+},\right.} & \left.\mathcal{K}_{m}^{\mathrm{cf}}\right]_{\mathbb{A}^{1}} \\
& \rightarrow\left[\Sigma_{s}^{p} \Sigma_{T} \wedge X_{+}, \mathcal{K}_{m}^{\mathrm{cf}}\right]_{\mathbb{A}^{1}} \rightarrow\left[\Sigma_{s}^{p}\left(\mathbb{P}_{X}^{1}\right)_{+}, \mathcal{K}_{m}^{\mathrm{cf}}\right]_{\mathbb{A}^{1}} .
\end{aligned}
$$

It follows from (1) and the first part of (2) that the first map in this exact sequence is surjective and the last term is zero if $0 \leq p<m$. Hence the third term must be zero.

Recall the ring isomorphism $K_{0}(\mathbb{C})[t] /(t-1)^{2} \simeq K_{0}\left(\mathbb{P}_{\mathbb{C}}^{1}\right)$. By Lemma 7.2.1, the element $(t-1)=([\mathcal{O}(1)]-[\mathcal{O}])$ defines a unique element th $\in\left[S^{1} \wedge T, \mathcal{K}_{1}^{\mathrm{cf}}\right]_{\mathbb{A}^{1}}$, called the Thom class. Since $S^{1} \wedge T$ is cofibrant and $\mathcal{K}_{1}^{\mathrm{cf}}$ is motivic fibrant, this yields a morphism $S^{1} \wedge T \rightarrow \mathcal{K}_{1}^{\mathrm{cf}}$ in $\mathbf{S p c}_{\bullet}(\mathbb{C})$, thus a morphism $\theta: T \rightarrow \Omega_{s} \mathcal{K}_{1}^{\mathrm{cf}}$ in $\mathbf{S p c}_{\bullet}(\mathbb{C})$. 
Definition 7.2.2 Define $\mathcal{K}^{\mathrm{alg}}=\left\{\mathcal{K}_{m, n}^{\mathrm{alg}}\right\} \in \operatorname{Spt}_{(s, \mathfrak{p})}(\mathbb{C})$ as $\left(\mathcal{K}^{\mathrm{cf}}, \Omega_{s}^{1} \mathcal{K}^{\mathrm{cf}}\{1\}, \Omega_{s}^{2} \mathcal{K}^{\mathrm{cf}}\{2\}, \ldots\right)$ with the following bonding maps: for $A \in \mathbf{S p t}(\mathbb{C}), B \in \mathbf{S p c}_{\bullet}(\mathbb{C})$, apply the map $\Omega_{S} A \wedge B \rightarrow \Omega_{S}(A \wedge B)$ repeatedly to get the morphisms

$$
\begin{aligned}
\Omega_{s}^{n} \mathcal{K}^{\mathrm{cf}}\{n\} \wedge T \rightarrow \Omega_{s}^{n}\left(\mathcal{K}^{\mathrm{cf}}\{n\}\right. & \wedge T) \stackrel{\Omega_{s}^{n}(\mathrm{Id} \wedge \theta)}{\longrightarrow} \Omega_{s}^{n}\left(\mathcal{K}^{\mathrm{cf}}\{n\} \wedge \Omega_{s} \mathcal{K}_{1}^{\mathrm{cf}}\right) \\
& \rightarrow \Omega_{s}^{n+1}\left(\mathcal{K}^{\mathrm{cf}}\{n\} \wedge \mathcal{K}_{1}^{\mathrm{cf}}\right) \stackrel{\Omega_{s}^{n+1}(\mathrm{Id} \wedge \phi)}{\longrightarrow} \Omega_{s}^{n+1} \mathcal{K}^{\mathrm{cf}}\{n+1\} .
\end{aligned}
$$

Proposition 7.2.3 The $(s, \mathfrak{p})$-bispectrum $\mathcal{K}^{\text {alg }}$ on $\mathbf{S m}_{\mathbb{C}}$ is an $\mathbb{A}^{1}-B G$ motivic $\Omega_{T^{-}}$ bispectrum, and it represents the algebraic $K$-theory in $\mathcal{S H}(\mathbb{C})$.

Proof Since $\mathcal{K}_{*, n}^{\mathrm{alg}}=\Omega_{s}^{n} \mathcal{K}^{\mathrm{cf}}\{n\}$ for each $n \geq 0$, by Corollary 3.1 .8 we see $\mathcal{K}^{\text {alg }}$ satisfies the $\mathbb{A}^{1}-\mathrm{BG}$ property. To show that $\mathcal{K}^{\text {alg }}$ is a motivic $\Omega_{T}$-bispectrum, it suffices to show that each map $\mathcal{K}_{m, n}^{\mathrm{alg}} \rightarrow \Omega_{T} \mathcal{K}_{m, n+1}^{\mathrm{alg}}$ between motivic fibrant pointed motivic spaces is a motivic weak-equivalence. For this, it suffices to show using Corollary 3.1.8 and Lemma 3.1.2 that for $X \in \mathbf{S m}_{\mathbb{C}}$ and $p \geq 0$, the induced map

$$
\left[\Sigma_{s}^{p} X_{+}, \Omega_{s}^{n} \mathcal{K}_{m+n}^{\mathrm{cf}}\right]_{\mathbb{A}^{1}} \rightarrow\left[\Sigma_{s}^{p} X_{+}, \Omega_{T} \Omega_{s}^{n+1} \mathcal{K}_{m+n+1}^{\mathrm{cf}}\right]_{\mathbb{A}^{1}}
$$

is an isomorphism, or that the map

$$
\left[\Sigma_{s}^{p} X_{+}, \Omega_{s}^{n} \mathcal{K}_{m+n}^{\mathrm{cf}}\right]_{\mathbb{A}^{1}} \rightarrow\left[\Sigma_{s}^{p} \Sigma_{T} X_{+}, \Omega_{s}^{n+1} \mathcal{K}_{m+n+1}^{\mathrm{cf}}\right]_{\mathbb{A}^{1}}
$$

is an isomorphism. By Corollary 3.1.8, this is equivalent to the fact that

$$
\left[\Sigma_{s}^{p} X_{+}, \mathcal{K}_{m}^{\mathrm{cf}}\right]_{\mathbb{A}^{1}} \rightarrow\left[\Sigma_{s}^{p} \Sigma_{T} X_{+}, \mathcal{K}_{m}^{\mathrm{cf}}\right]_{\mathbb{A}^{1}}
$$

is an isomorphism. But, by Lemma 7.2.1 and the definition of the Thom class, for $0 \leq p<m$ both terms are zero, while for $p \geq m \geq 0$ this map is just the multiplication by the Thom class on the groups $K_{p-m}(X) \rightarrow K_{p-m}\left(\mathbb{P}_{X}^{1},\{\infty\} \times X\right)$. This is an isomorphism by the projective bundle formula. The representability now follows using Theorems 3.3.3, 7.1.1 and Corollary 3.1.6.

Theorem 7.2.4 Let $X \in \mathbf{S m}_{\mathbb{C}}$ and $p \in \mathbb{Z}$. We have an isomorphism

$$
K_{p}^{\text {sst }}(X) \simeq\left[\Sigma_{T}^{\infty} \Sigma_{s}^{\infty} X_{+}[p],\left(\mathcal{K}^{\text {alg }}\right)^{\text {host }}\right]_{\mathbb{A}^{1}},
$$

ie the semitopological $K$-theory is representable in $\mathcal{S H}(\mathbb{C})$. 
Proof By Theorems 6.2.1, 6.2.3 and Proposition 7.2.3, we may replace $\left(\mathcal{K}^{\text {alg }}\right)^{\text {host }}$ in the above by $\left(\mathcal{K}^{\mathrm{alg}}\right)^{\text {sst }}$. Let $f:\left(\mathcal{K}^{\mathrm{alg}}\right)^{\text {sst }} \rightarrow F$ be a stable motivic fibrant replacement of $\left(\mathcal{K}^{\text {alg }}\right)^{\text {sst }}$. This $F=\left(F_{0}, F_{1}, \ldots\right)$ is a $T$-levelwise motivic fibrant motivic $\Omega_{T^{-}}$ bispectrum. We have isomorphisms

$$
\begin{aligned}
{\left[\Sigma_{T}^{\infty} \Sigma_{s}^{\infty} X_{+}[p],\left(\mathcal{K}^{\mathrm{alg}}\right)^{\mathrm{sst}}\right]_{\mathbb{A}^{1}} } & \simeq\left[\Sigma_{T}^{\infty} \Sigma_{s}^{\infty} X_{+}[p], F\right]_{\mathbb{A}^{1}} \\
& \simeq\left[\Sigma_{s}^{\infty} X_{+}[p], \Omega_{T}^{\infty} F\right]_{\mathbb{A}^{1}}=\left[\Sigma_{S}^{\infty} X_{+}[p], F_{0}\right]_{\mathbb{A}^{1}} .
\end{aligned}
$$

By Theorems 3.3.3 and 6.2.1 and Proposition 7.2.3, the map $f$ is a $T$-levelwise objectwise weak-equivalence. In particular, the map $\left(\mathcal{K}_{*, 0}^{\text {alg }}\right)^{\text {sst }}=\left(\mathcal{K}^{\text {alg }}\right)_{*, 0}^{\text {sst }} \rightarrow F_{0}$ is an objectwise weak-equivalence, so

$$
\left[\Sigma_{s}^{\infty} X_{+}[p], F_{0}\right]_{\mathbb{A}^{1}} \simeq\left[\Sigma_{s}^{\infty} X_{+}[p],\left(\mathcal{K}_{*, 0}^{\text {alg }}\right)^{\text {sst }}\right]_{\mathbb{A}^{1}} \simeq\left[\Sigma_{s}^{\infty} X_{+}[p],\left(\mathcal{K}^{\text {cf }}\right)^{\text {sst }}\right]_{\mathbb{A}^{1}} .
$$

By Theorems 3.1.5, 5.1.5 and 7.1.1, we have that the maps $\mathcal{K}^{\text {sst }} \rightarrow\left(\mathcal{K}^{\text {fib }}\right)^{\text {sst }} \leftarrow\left(\mathcal{K}^{\text {cf }}\right)^{\text {sst }}$ are objectwise weak-equivalences, so the last group is $\left[\Sigma_{s}^{\infty} X_{+}[p], \mathcal{K}^{\text {sst }}\right]_{\mathbb{A}^{1}}$. But, by Proposition 7.1.2, this is $K_{p}^{\text {sst }}(X)$.

\section{Representing morphic cohomology in $\mathcal{S H}(\mathbb{C})$}

The morphic cohomology $L^{p} H^{q}(X)$ for smooth quasiprojective schemes $X$ over $\mathbb{C}$ was introduced in [5], as the homotopy groups of a function space. Later it was identified in Friedlander and Walker [10] as the homotopy group of the semitopologization of the complex of Friedlander and Suslin. We show that the morphic cohomology is representable in $\mathcal{S H}(\mathbb{C})$ by homotopy semitopologizing the motivic EilenbergMac Lane spectrum of Voevodsky.

\subsection{Motivic Eilenberg-Mac Lane spectrum}

Recall (see [7, page 141] and Mazza, Voevodsky and Weibel [27, page 126]) the following. Let $r \geq 0$ and let $f: Z \rightarrow U$ be a morphism, where each irreducible component of $Z$ dominates a component of $U$. We say $Z$ is equidimensional of relative dimension $r$ over $U$ if for every $s \in U$, the scheme-theoretic fiber $Z_{s}$ is either $\varnothing$ or an equidimensional of dimension $r$. For $X \in \mathbf{S c h}_{\mathbb{C}}$ and $U \in \mathbf{S m}_{\mathbb{C}}$, let $z_{\text {equi }}(X, r)(U)$ be the group of cycles on $Z$ of $X \times U$ that are dominant and equidimensional of relative dimension $r$ over a component of $U$. This $z_{\text {equi }}(X, r)$ is a presheaf (in fact an étale sheaf) on $\mathbf{S m}_{\mathbb{C}}$. Let $\Delta^{\bullet}$ be the cosimplicial scheme, where $\Delta^{n}=$ $\operatorname{Spec}\left(\mathbb{C}\left[t_{0}, \ldots, t_{n}\right]\right) /\left(\sum_{i=0}^{n} t_{i}-1\right)$ and $\partial_{i}^{n}(0 \leq i \leq n)$ are the cofaces. For $U \in \mathbf{S m}_{\mathbb{C}}$, and a presheaf $F$ of abelian groups on $\mathbf{S m}_{\mathbb{C}}$, the simplicial abelian group $F\left(\Delta^{\bullet} \times U\right)$ has its associated chain complex $\underline{C}_{*} F(U)$, namely, $\underline{C}_{n} F(U)=F\left(\Delta^{n} \times U\right)$ with the 
differential $\sum_{i=0}^{n}(-1)^{i} F\left(\partial_{i}^{n} \times \operatorname{Id}_{U}\right)$. This $\underline{C}_{*} F$ is a presheaf of chain complexes of abelian groups on $\mathbf{S m}_{\mathbb{C}}$. For $n \geq 0$, the Friedlander-Suslin motivic complex $\mathbb{Z}^{\mathrm{FS}}(n)$ on $\mathbf{S m}_{\mathbb{C}}$ is $\underline{C}_{*} z_{\text {equi }}\left(\mathbb{A}^{n}, 0\right)$. (This definition of $\mathbb{Z}^{\mathrm{FS}}(n)$ differs slightly from the one in [27], where $\mathbb{Z}^{\mathrm{FS}}(n)$ is defined as $\underline{C}_{*} z_{\text {equi }}\left(\mathbb{A}^{n}, 0\right)[-2 n]$.) In what follows, we identify the presheaf $\mathbb{Z}^{\mathrm{FS}}(n)$ with an object of Spc.( $\left.\mathbb{C}\right)$ via the Dold-Kan correspondence. Recall (see [41, Section 6.1]) that the motivic Eilenberg-Mac Lane spectrum $\mathbf{H Z}$ is a sequence of pointed simplicial presheaves, whose $n^{\text {th }}$ level is $K(\mathbb{Z}(n), 2 n)=\underline{C}_{*} L\left(T^{n}\right)$ for some functor $L$, with motivic weak-equivalences

$$
K(\mathbb{Z}(n), 2 n) \rightarrow \Omega_{T} K(\mathbb{Z}(n+1), 2 n+2) .
$$

For $X \in \mathbf{S m}_{\mathbb{C}}$ and $U \in \mathbf{S m}_{\mathbb{C}}, L(X)(U)$ is the group of cycles on $U \times X$, finite over $U$ and surjective over a connected component of $U$. This $L(X)$ is a presheaf on $\mathbf{S m}_{\mathbb{C}}$. This $L$ even extends to $\mathbf{S p c}_{\bullet}(\mathbb{C})$. Using the isomorphisms $T^{n} \simeq \mathbb{P}^{n} / \mathbb{P}^{n-1}$ and $\underline{C}_{*} L(A / B) \simeq \underline{C}_{*} L(A) / \underline{C}_{*} L(B)$, we see that $K(\mathbb{Z}(n), 2 n) \simeq$ $\underline{C}_{*} L\left(\mathbb{P}^{n}\right) / \underline{C}_{*} L\left(\mathbb{P}^{n-1}\right)$, which is isomorphic (via localization and Dold and Kan) to the presheaf $\underline{C}_{*} z_{\text {equi }}\left(\mathbb{A}^{n}, 0\right)=\mathbb{Z}^{\mathrm{FS}}(n)$ of complexes seen as an object in Spc $\mathbf{S}(\mathbb{C})$. Thus, $\mathbf{H} \mathbb{Z}$ can be regarded as the motivic $T$-spectrum $\left(\mathbb{Z}^{\mathrm{FS}}(0), \mathbb{Z}^{\mathrm{FS}}(1), \ldots\right)$.

\section{2 $\mathbb{A}^{1}-B G$ property $H \mathbb{Z}$}

For $\mathbb{Z}^{\mathrm{FS}}(n)$, the $\mathbb{A}^{1}$-weak-invariance holds by [27, Corollary 2.19], while the BG property follows from Suslin and Voevodsky [39, Proposition 4.3.9] combined with the proof of the Zariski Mayer-Vietoris property in [7, Theorem 5.11]. Thus:

Proposition 8.2.1 The sheaves $\mathbb{Z}^{\mathrm{FS}}(n)$ satisfy the $\mathbb{A}^{1}-B G$ property on $\mathbf{S m}_{\mathbb{C}}$.

Recall from Section 2.3.2 that for a $T$-spectrum $E$, the associated $(s, \mathfrak{p})$-bispectrum $E$ is given by $\Sigma_{s}^{\infty} E=\left(\Sigma_{s}^{\infty} E_{0}, \Sigma_{s}^{\infty} E_{1}, \ldots\right)$.

Proposition 8.2.2 The $(s, \mathfrak{p})$-bispectrum $\Sigma_{s}^{\infty} \mathbf{H} \mathbb{Z}$ satisfies the following properties.

(1) It is a $T$-levelwise objectwise $\Omega_{s}$-spectrum, ie $\Sigma_{S}^{\infty} \mathbb{Z}^{\mathrm{FS}}(n)$ is an objectwise $\Omega_{s}$-spectrum for each $n \geq 0$.

(2) It is an $S^{1}$-levelwise motivic $\Omega_{T}$-spectrum, ie $\Sigma_{S}^{n} \mathbf{H} \mathbb{Z}$ is a motivic $\Omega_{T^{-}}$ spectrum for each $n \geq 0$.

(3) It satisfies the $\mathbb{A}^{1}-B G$ property.

(4) The properties (1)-(3) also hold for $\left(\Sigma_{S}^{\infty} \mathbf{H} \mathbb{Z}\right)^{\text {sst }}$. 
Proof For a simplicial abelian group $A$ and $K \in \mathbf{S p c}$, there is a simplicial abelian group $K \otimes A$, given by $\mathbb{Z}\left[K_{n}\right] \otimes_{\mathbb{Z}} A_{n}$ at level $n$, where $\mathbb{Z}\left[K_{n}\right]$ is the free abelian group on $K_{n}$. The pointed motivic space $S^{1} \wedge \mathbb{Z}^{\mathrm{FS}}(n)$ corresponds to the presheaf $S^{1} \otimes \mathbb{Z}^{\mathrm{FS}}(n)$ of simplicial abelian groups under Dold-Kan correspondence. It follows from Goerss and Jardine [12, Lemma 4.53] that $\Sigma_{s}^{\infty} \mathbb{Z}^{\mathrm{FS}}(n)$ is an objectwise $\Omega_{s}$-spectrum. This proves (1). Part (2) follows from [41, Theorem 6.2] and the facts that $\Sigma_{s}$ preserves motivic weak-equivalences and that the map $\Sigma_{S}\left(\Omega_{T} E\right) \rightarrow \Omega_{T}\left(\Sigma_{S} E\right)$ is an objectwise weakequivalence for $E \in \mathbf{S p c}_{\bullet}(\mathbb{C})$. Part (3) is equivalent to the fact that $\Sigma_{s}^{\infty} \mathbb{Z}^{\mathrm{FS}}(n)$ is an $\mathbb{A}^{1}-$ BG presheaf of spectra. This follows from Proposition 8.2.1, part (1), Corollary 3.2.4 and Theorem 3.1.5. For (4), the $\mathbb{A}^{1}-B G$ property of $\left(\Sigma_{s}^{\infty} \mathbf{H} \mathbb{Z}\right)^{\text {sst }}$ follows from part (3) and Theorem 6.1.5. Furthermore, Proposition 8.2.1 and Theorem 6.1.5 show that each $\left(\mathbb{Z}^{\mathrm{FS}}(n)\right)^{\text {sst }}$ is $\mathbb{A}^{1}-\mathrm{BG}$. We deduce from [12, Lemma 4.53] that $\Sigma_{s}^{\infty}\left(\mathbb{Z}^{\mathrm{FS}}(n)\right)^{\text {sst }}$ is an objectwise $\Omega_{s}$-spectrum. The isomorphism $\left(\Sigma_{s}(-)\right)^{\text {sst }} \simeq \Sigma_{s}(-)^{\text {sst }}$ now implies that $\left(\Sigma_{s}^{\infty} \mathbf{H} \mathbb{Z}\right)^{\text {sst }}$ is a $T$-levelwise objectwise $\Omega_{s}$-spectrum. That it is an $\Omega_{T}$-bispectrum follows from part (2) and Theorem 6.2.1(2).

For $E=\left(E_{0}, E_{1}, \ldots\right) \in \mathbf{S p t}_{(s, \mathfrak{p})}(\mathbb{C})$, with $E_{i} \in \mathbf{S p t}(\mathbb{C}), E\{m\} \in \mathbf{S p t}_{(s, \mathfrak{p})}(\mathbb{C})$ is $\left(E_{m}, E_{m+1}, \ldots\right)$. By [15, Lemma 3.8, Theorem 3.9], $s_{-}: E \mapsto E\{1\}$ is a right Quillen endofunctor on $\mathbf{S p t}_{(s, \mathfrak{p})}(\mathbb{C})$ and we have isomorphisms of functors $\Sigma_{T} \simeq \boldsymbol{L}_{T} \simeq \boldsymbol{R} s_{-}$ on $\mathcal{S H}(\mathbb{C})$. Recall (Section 2.3.2) that there are adjoint functors $\Sigma_{T}^{\infty}: \mathcal{S H}_{S^{1}}(\mathbb{C}) \leftrightarrow$ $\mathcal{S H}(\mathbb{C}): \boldsymbol{R} \Omega_{T}^{\infty}$.

Corollary 8.2.3 In $\mathcal{S H} \mathcal{H}^{1}(\mathbb{C})$, we have $\Sigma_{S}^{\infty}\left(\mathbb{Z}^{\mathrm{FS}}(n)\right)^{\text {sst }} \simeq \boldsymbol{R} \Omega_{T}^{\infty} \Sigma_{T}^{n}\left(\Sigma_{S}^{\infty} \mathbf{H} \mathbb{Z}\right)^{\text {host }}$.

Proof Let $f:\left(\Sigma_{s}^{\infty} \mathbf{H} \mathbb{Z}\right)^{\text {sst }} \rightarrow F$ be a stable motivic fibrant replacement. We have, by Proposition 8.2.2 and Theorem 6.2.1, that $f$ is $T$-levelwise objectwise weakequivalence of $\mathbb{A}^{1}-\mathrm{BG}(s, \mathfrak{p})$-bispectra. This implies

$$
\Sigma_{T}^{n}\left(\Sigma_{s}^{\infty} \mathbf{H} \mathbb{Z}\right)^{\text {host }} \simeq \boldsymbol{R}^{n} s_{-}\left(\Sigma_{s}^{\infty} \mathbf{H} \mathbb{Z}\right)^{\text {host }} \simeq F\{n\} \simeq\left(\Sigma_{s}^{\infty} \mathbf{H} \mathbb{Z}\right)^{\text {sst }}\{n\} \simeq\left(\Sigma_{s}^{\infty} \mathbf{H} \mathbb{Z}\{n\}\right)^{\text {sst }} .
$$

Applying Proposition 8.2.2 and Theorem 6.2.1 once again,

$$
\begin{aligned}
\boldsymbol{R} \Omega_{T}^{\infty} \Sigma_{T}^{n}\left(\Sigma_{s}^{\infty} \mathbf{H} \mathbb{Z}\right)^{\mathbf{h o s t}} \simeq \Omega_{T}^{\infty}\left(\left(\Sigma_{s}^{\infty} \mathbf{H} \mathbb{Z}\{n\}\right)^{\text {sst }}\right) & \simeq \operatorname{Ev}_{0}\left(\left(\Sigma_{s}^{\infty} \mathbf{H} \mathbb{Z}\{n\}\right)^{\text {sst }}\right) \\
& \simeq\left(\Sigma_{s}^{\infty} \mathbb{Z}^{\mathrm{FS}}(n)\right)^{\text {sst }} .
\end{aligned}
$$

Since $\left(\Sigma_{S}(-)\right)^{\text {sst }} \simeq \Sigma_{S}(-)^{\text {sst }}$, the corollary follows.

Corollary 8.2.4 In $\mathcal{S H}(\mathbb{C})$, we have $\left(\Sigma_{T}^{n} \Sigma_{S}^{\infty} \mathbf{H} \mathbb{Z}\right)^{\text {host }} \simeq \Sigma_{T}^{n}\left(\Sigma_{s}^{\infty} \mathbf{H} \mathbb{Z}\right)^{\text {host }}$.

Proof Under the notation of the proof of Corollary 8.2.3, we get $\left(\Sigma_{T}^{n} \Sigma_{S}^{\infty} \mathbf{H} \mathbb{Z}\right)^{\text {host }} \simeq$ $\left(\boldsymbol{R}^{n} s_{-} \Sigma_{s}^{\infty} \mathbf{H} \mathbb{Z}\right)^{\text {host }}$. Here, this is isomorphic to $\left(\Sigma_{s}^{\infty} \mathbf{H} \mathbb{Z}\{n\}\right)^{\text {host }}$ by Proposition 8.2.2 and Theorem 3.3.3. This equals $\left(\Sigma_{s}^{\infty} \mathbf{H} \mathbb{Z}\{n\}\right)^{\text {sst }}$ by Proposition 8.2.2. But, in the proof of Corollary 8.2.3, we saw this is $\Sigma_{T}^{n}\left(\Sigma_{S}^{\infty} \mathbf{H} \mathbb{Z}\right)^{\text {host }}$. 
Theorem 8.2.5 Let $X$ be a smooth quasiprojective scheme over $\mathbb{C}$ and let $n \geq 0$ and $p \in \mathbb{Z}$. Then $L^{n} H^{2 n-p}(X) \simeq\left[\Sigma_{T}^{\infty} \Sigma_{S}^{\infty} X_{+}[p], \Sigma_{T}^{n}\left(\Sigma_{S}^{\infty} \mathbf{H} \mathbb{Z}\right)^{\text {host }}\right]_{\mathbb{A}^{1}}$. That is, the morphic cohomology of smooth quasiprojective schemes is representable in $\mathcal{S H}(\mathbb{C})$.

Proof We have

$$
\begin{aligned}
{\left[\Sigma_{T}^{\infty} \Sigma_{s}^{\infty} X_{+}[p], \Sigma_{T}^{n}\left(\Sigma_{s}^{\infty} \mathbf{H} \mathbb{Z}\right)^{\text {host }}\right]_{\mathbb{A}^{1}} } & \simeq\left[\Sigma_{s}^{\infty} X_{+}[p], \boldsymbol{R} \Omega_{T}^{\infty} \Sigma_{T}^{n}\left(\Sigma_{s}^{\infty} \mathbf{H} \mathbb{Z}\right)^{\text {host }}\right]_{\mathbb{A}^{1}} \\
& \simeq\left[\Sigma_{s}^{\infty} X_{+}[p], \Sigma_{s}^{\infty}\left(\mathbb{Z}^{\mathrm{FS}}(n)\right)^{\text {sst }}\right]_{\mathbb{A}^{1}}
\end{aligned}
$$

by adjointness and Corollary 8.2.3. This is isomorphic to $\pi_{p}\left(\Sigma_{s}^{\infty}\left(\mathbb{Z}^{\mathrm{FS}}(n)\right)^{\mathrm{sst}}(X)\right)$ by Proposition 8.2.2 and Corollary 3.1.6, which in turn is equal to $\pi_{p}\left(\left(\mathbb{Z}^{\mathrm{FS}}(n)\right)^{\mathrm{sst}}(X)\right)$ by Proposition 8.2.2. This last group is $L^{n} H^{2 n-p}(X)$ by [10, Corollary 3.5]. This proves the result.

Remark 8.2.6 Chu [3] proves that the morphic cohomology is representable in the Voevodsky $\mathcal{D} \mathcal{M}(\mathbb{C})$ of motives. Using motivic symmetric spectra (MSS) of Jardine [18] as a model for $\mathcal{S H}(\mathbb{C})$, Röndigs and Østvær [36] identified $\mathcal{H}\left(\mathrm{MSS}^{\mathrm{tr}}\right)$ (MSS with trace) with $\mathcal{D} \mathcal{M}(\mathbb{C})$, and constructed a Dold-Kan map $\psi: \mathcal{H}\left(\mathrm{MSS}^{\text {tr }}\right) \rightarrow \mathcal{H}(\mathrm{MSS})$ to give adjoint functors $\phi: \mathcal{S H}(\mathbb{C}) \rightleftharpoons \mathcal{D} \mathcal{M}(\mathbb{C}): \psi$. By construction, one can check that $\phi\left(\left(\Sigma_{T}^{n} \Sigma_{s}^{\infty} \mathbf{H} \mathbb{Z}\right)^{\text {host }}\right)$ is Chu's $\wp_{\text {mor }}(n)$ and our result is compatible with Chu's.

\subsection{Excision and localization for morphic cohomology}

As a consequence of Theorems 7.2.4 and 8.2.5, we obtain the following:

Theorem 8.3.1 The morphic cohomology of smooth schemes over $\mathbb{C}$ satisfies Nisnevich descent and localization.

Proof The arguments are standard, so we sketch the ideas. Given a Nisnevich square as in (3.0.1), by [34, Corollary 2.20] there is a distinguished triangle in $\mathcal{S H}(\mathbb{C})$ of the form $\Sigma_{T}^{\infty} \Sigma_{S}^{\infty} W_{+} \rightarrow \Sigma_{T}^{\infty} \Sigma_{S}^{\infty} U_{+} \vee \Sigma_{T}^{\infty} \Sigma_{S}^{\infty} V_{+} \rightarrow \Sigma_{T}^{\infty} \Sigma_{S}^{\infty} X_{+} \rightarrow \Sigma_{T}^{\infty} \Sigma_{S}^{\infty} W_{+}[1]$. By applying $\left[-,\left(\Sigma_{S}^{\infty} \mathbf{H} \mathbb{Z}\right)^{\text {host }}\right]_{\mathbb{A}^{1}}$ and $\left[-,\left(\mathcal{K}^{\text {alg }}\right)^{\text {host }}\right]_{\mathbb{A}^{1}}$, we obtain Nisnevich descent property. For localization, given a smooth closed immersion $Z \hookrightarrow X$ and the open complement $U \subset X$, by [34, Lemma 2.16, Theorem 2.26] we have a distinguished triangle in $\mathcal{S H}(\mathbb{C})$ of the form $\Sigma_{T}^{\infty} \Sigma_{S}^{\infty} U_{+} \rightarrow \Sigma_{T}^{\infty} \Sigma_{S}^{\infty} X_{+} \rightarrow \Sigma_{T}^{\infty} \Sigma_{S}^{\infty} \mathrm{Th}\left(N_{Z / X}\right) \rightarrow$ $\Sigma_{T}^{\infty} \Sigma_{s}^{\infty} U_{+}[1]$, where $\operatorname{Th}\left(N_{Z / X}\right)$ is the Thom space of the normal bundle. Applying $\left[-,\left(\Sigma_{S}^{\infty} \mathbf{H} \mathbb{Z}\right)^{\text {host }}\right]_{\mathbb{A}^{1}}$ and $\left[-,\left(\mathcal{K}^{\text {alg }}\right)^{\text {host }}\right]_{\mathbb{A}^{1}}$ again, we obtain localization sequences, provided Thom isomorphisms of cohomologies of $Z$ and $\operatorname{Th}\left(N_{Z / X}\right)$, up to a shift. Then the projective bundle formula gives Chern classes (see Panin [35, Section 3.6]), and Thom isomorphism by [35, Theorem 3.35]. 
Remark 8.3.2 The definitions of $L^{p} H^{q}$ in $[5 ; 10]$ assume quasiprojectivity of the underlying scheme, but we can redefine the morphic cohomology for all $X \in \mathbf{S m}_{\mathbb{C}}$ using host, as $L^{n} H^{2 n-p}(X):=\left[\Sigma_{T}^{\infty} \Sigma_{S}^{\infty} X_{+}[p], \Sigma_{T}^{n}\left(\Sigma_{S}^{\infty} \mathbf{H} \mathbb{Z}\right)^{\text {host }}\right]_{\mathbb{A}^{1}}$. By Theorem 8.2.5, this coincides with the previous one.

\section{Semitopological cobordism}

The motivic Thom spectrum MGL (see [41, Section 6.3]) is a $T$-spectrum

$$
\left(\mathrm{MGL}_{0}, \mathrm{MGL}_{1}, \ldots\right) \text {, }
$$

where $\mathrm{MGL}_{n}$ is the motivic Thom space of the universal rank- $n$ vector bundle $E_{n}$ on the Grassmann ind-scheme $\operatorname{Gr}(n, \infty)$. The associated cohomology theory (Section 2.3.3) $\mathrm{MGL}^{p, q}(-)$ on $\mathbf{S m}_{\mathbb{C}}$ is called the (Voevodsky) algebraic cobordism.

As an application of Theorem 6.2.3, we can define the semitopological Thom spectrum $\mathrm{MGL}_{\text {sst }}$ to be MGL ${ }^{\text {host }}$ in $\mathcal{S H}(\mathbb{C})$. We call its associated bigraded cohomology theory $\operatorname{MGL}_{\mathbf{s s t}}^{p, q}(-)$ on $\mathbf{S m}_{\mathbb{C}}$, the semitopological cobordism. The natural map MGL $\rightarrow$ MGL $_{\text {sst }}$ in $\mathcal{S H}(\mathbb{C})$ defines a natural transformation of bigraded cohomology theories $\mathrm{MGL}^{p, q}(-) \rightarrow \mathrm{MGL}_{\mathrm{sst}}^{p, q}(-)$ on $\mathbf{S m}_{\mathbb{C}}$. Using the morphism MGL $\rightarrow \boldsymbol{H} \mathbb{Z}$, it follows from Theorem 8.2.5 that there is a commutative diagram

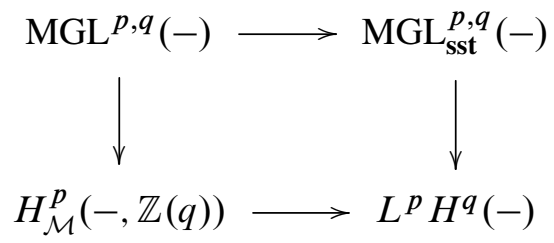

of cohomology theories on $\mathbf{S m}_{\mathbb{C}}$. (The referee had kindly informed that J Heller [13] had earlier defined this semitopological cobordism by taking a fibrant replacement of MGL and applying the sst-functor. By motivic descent theorems in Section 3 and Theorem 6.1.5, this is objectwise weak-equivalent to ours, so that the resulting cohomology theories are equal.) A result of Hopkins and Morel says that, for $X \in \mathbf{S m}_{\mathbb{C}}$ and $n \geq 0$, there is an Atiyah-Hirzebruch-type spectral sequence

$$
E^{p, q}(n)=H_{\mathcal{M}}^{p-q}(X, \mathbb{Z}(n-q)) \otimes_{\mathbb{Z}} \mathbb{L}^{q} \Rightarrow \operatorname{MGL}^{p+q, n}(X),
$$

where $\mathbb{L}=\bigoplus_{q \leq 0} \mathbb{L}^{q}$ is the Lazard ring. This result is in an unpublished form to the best of our knowledge, but based on the lecture notes in Lawson [22], a proof of an essential part is done in Hoyois [16]. Our last goal is to apply host and the ideas of [16], Spitzweck [37; 38] and Voevodsky [42] to produce an analogous spectral sequence for 
$\mathrm{MGL}_{\text {sst }}$. We remark that a similar spectral sequence that relates the motivic cohomology to the algebraic $K$-theory was constructed in Bloch and Lichtenbaum [1] and [6], while for the semitopological $K$-theory in Friedlander, Haesemeyer and Walker [4].

Recall from [38, Section 3] an analogue of the Postnikov tower for $E \in \mathcal{S H}(\mathbb{C})$. Let $\mathcal{S H}(\mathbb{C})^{\text {eff }} \subset \mathcal{S H}(\mathbb{C})$ be the full localizing triangulated subcategory generated by $\Sigma_{s}^{i} \Sigma_{t}^{j} \Sigma_{T}^{\infty} X_{+}$for $i, j \in \mathbb{Z}, j \geq 0$ and $X \in \mathbf{S m}_{\mathbb{C}}$. For $p \in \mathbb{Z}$, the inclusion

$$
\iota_{p}: \Sigma_{T}^{p} \mathcal{S H}(\mathbb{C})^{\mathrm{eff}} \rightarrow \mathcal{S H}(\mathbb{C})
$$

has a right adjoint $r_{p}$ such that $r_{p} \circ \iota_{p} \simeq \operatorname{Id~(cf~[34,~Section~4]).~Set~} f_{p}:=\iota_{p} \circ r_{p}$. There is a natural transformation $\rho_{p+1}: f_{p+1} \rightarrow f_{p}$. We define the slices $s_{p} E:=\operatorname{cofib}\left(\rho_{p+1}\right)$. Thus, we have a sequence of maps $\rightarrow f_{p} E \rightarrow \cdots \rightarrow f_{1} E \rightarrow f_{0} E \rightarrow f_{-1} E \rightarrow \cdots \rightarrow E$. We also have a distinguished triangle $f_{p+1} E \rightarrow f_{p} E \rightarrow s_{p} E \rightarrow\left(f_{p+1} E\right)[1]$ in $\mathcal{S H}(\mathbb{C})$.

We say that $E$ is effective if the map $f_{p} E \rightarrow E$ is an isomorphism for $p \leq 0$. By [34, Remark 4.2; 37, Corollary 3.2] we have $f_{p}$ MGL $\simeq$ MGL for all $p \leq 0$ and $s_{p} \mathrm{MGL}=0$ for all $p<0$. In particular, MGL is effective. For $s_{0}$ MGL, the natural map MGL $\rightarrow \mathbf{H Z}$ induces an isomorphism $s_{0} \mathrm{MGL} \simeq \mathbf{H} \mathbb{Z}$, by combining [37, Corollary 3.3; 42]. Recall there is a morphism of ring spectra $\mathbb{L} \rightarrow$ MGL and the natural map $\mathrm{MGL} \rightarrow s_{0} \mathrm{MGL}=\mathbf{H} \mathbb{Z}$ factors as MGL $\rightarrow \mathrm{MGL} \otimes_{\mathbb{L}}\left(\mathbb{L} / \mathbb{L}^{<0}\right)=\mathrm{MGL} \otimes_{\mathbb{L}} \mathbb{Z} \rightarrow \mathbf{H Z}$. The last map is an isomorphism in $\mathcal{S H}(\mathbb{C})$ by [16]. This implies $s_{p}$ MGL $\stackrel{\sim}{\rightarrow} \Sigma_{T}^{p} \mathbf{H} \mathbb{L}^{p}$ by [37, Theorem 4.7], which we use below.

Fix $X \in \mathbf{S m}_{\mathbb{C}}$ and $n \geq 0$. We write $\Sigma_{T}^{\infty} X_{+}$as just $X$ and the hom sets $[-,-]_{\mathbb{A}^{1}}$ in $\mathcal{S H}(\mathbb{C})$ as just $[-,-]$. Applying host to the sequence

$$
\rightarrow f_{2} \mathrm{MGL} \rightarrow f_{1} \mathrm{MGL} \rightarrow f_{0} \mathrm{MGL}=\mathrm{MGL}
$$

and the distinguished triangle

$$
f_{p+1} \mathrm{MGL} \rightarrow f_{p} \mathrm{MGL} \rightarrow s_{p} \mathrm{MGL} \rightarrow\left(f_{p+1} \mathrm{MGL}\right)[1],
$$

we get the sequences of maps

$$
\cdots \rightarrow\left(f_{p} \mathrm{MGL}\right)^{\text {host }} \rightarrow \cdots \rightarrow\left(f_{1} \text { MGL }\right)^{\text {host }} \rightarrow\left(f_{0} \text { MGL }\right)^{\text {host }}=\text { MGL }_{\text {sst }},
$$

and by Theorem 6.2.3 a distinguished triangle

$$
\left(f_{p+1} \mathrm{MGL}\right)^{\text {host }} \rightarrow\left(f_{p} \mathrm{MGL}\right)^{\text {host }} \rightarrow\left(s_{p} \text { MGL }\right)^{\text {host }} \rightarrow\left(f_{p+1} \text { MGL }\right)^{\text {host }}[1]
$$

in $\mathcal{S H}(\mathbb{C})$. Applying $[X,-]$ to the triangle, we obtain an exact sequence

$$
\begin{aligned}
{\left[X,\left(f_{p+1} \mathrm{MGL}\right)^{\text {host }}\right] \rightarrow\left[X,\left(f_{p} \mathrm{MGL}\right)^{\text {host }}\right] \rightarrow[} & \left.X,\left(s_{p} \text { MGL }\right)^{\text {host }}\right] \\
& \rightarrow\left[X,\left(f_{p+1} \text { MGL }\right)^{\text {host }}[1]\right]
\end{aligned}
$$


We now construct some exact couples. See McCleary [28, Section 2, Theorem 2.8] for related formalisms. For $p, q \in \mathbb{Z}$ and $n \geq 0$, define

$$
A^{p, q}(X, n):=\left[X, \Sigma_{s}^{p+q-n} \Sigma_{t}^{n}\left(f_{p} \text { MGL }\right)^{\text {host }}\right] .
$$

The map $\rho_{p}^{\text {host }}:\left(f_{p} \text { MGL }\right)^{\text {host }} \rightarrow\left(f_{p-1} \text { MGL }\right)^{\text {host }}$ induces a map

$$
\rho_{p-1, q+1}: A^{p, q}(X, n) \rightarrow A^{p-1, q+1}(X, n) .
$$

For the slices, we let $E^{p, q}(X, n):=\left[X, \Sigma_{S}^{p+q-n} \Sigma_{t}^{n}\left(s_{p} \text { MGL }\right)^{\text {host }}\right]$. From (9.0.2), we get an exact sequence

$$
A^{p, q}(X, n) \rightarrow A^{p-1, q+1}(X, n) \rightarrow E^{p-1, q+1}(X, n) \rightarrow A^{p+1, q}(X, n),
$$

where $\rho_{p-1, q+1}, \gamma_{p-1, q+1}$ and $\delta_{p-1, q+1}$ are the arrows. We set

$$
D_{1}(X, n):=\bigoplus_{p, q} A^{p, q}(X, n) \quad \text { and } \quad E_{1}(X, n):=\bigoplus_{p, q} E^{p, q}(X, n) .
$$

Write $a_{1}:=\bigoplus \delta_{p-1, q+1}, b_{1}:=\bigoplus \rho_{p-1, q+1}$ and $c_{1}:=\bigoplus \gamma_{p-1, q+1}$. This gives an exact couple $\left\{D_{1}, E_{1}, b_{1}, c_{1}, a_{1}\right\}$. We let $d_{1}:=c_{1} \circ a_{1}: E_{1} \rightarrow E_{1}$. That (9.0.2) is exact implies that $d_{1}^{2}=0$, and $\left(E_{1}, d_{1}\right)$ is a complex. Repeatedly taking homology, we obtain a spectral sequence. For the target of the spectral sequence, let $A^{m}(X, n):=\operatorname{colim}_{q \rightarrow \infty} A^{m-q, q}(X, n)$. Since $X$ is a compact object of $\mathcal{S H}(\mathbb{C})(\mathrm{cf}$ [41, Proposition 5.5]), the colimit enters into $[-,-]$ thus

$$
A^{m}(X, n)=\left[X, \Sigma_{s}^{m-n} \Sigma_{t}^{n} \mathrm{MGL}^{\text {host }}\right]=\operatorname{MGL}_{\mathrm{sst}}^{m, n}(X)
$$

by (9.0.1). The formalism of exact couples yields a spectral sequence

$$
E_{1}^{p, q}(X, n)=E^{p, q}(X, n) \Rightarrow A^{p+q}(X, n) .
$$

We have

$$
E_{1}^{p, q}(X, n) \simeq\left[X, \Sigma_{s}^{p+q-n} \Sigma_{t}^{n}\left(s_{p} \mathrm{MGL}\right)^{\mathbf{h o s t}}\right] \simeq\left[X, \Sigma_{s}^{p+q-n} \Sigma_{t}^{n}\left(\Sigma_{T}^{p} \mathbf{H} \mathbb{L}^{p}\right)^{\mathbf{h o s t}}\right]
$$

because $s_{p}$ MGL $\stackrel{\sim}{\rightarrow} \Sigma_{T}^{p} \mathbf{H} \mathbb{L}^{p}$ by [37, Theorem 4.7]. By Corollary 8.2.4 and adjointness, this is

$$
\begin{aligned}
{\left[X, \Sigma_{s}^{p+q-n} \Sigma_{t}^{n} \Sigma_{T}^{p}\left(\mathbf{H} \mathbb{L}^{p}\right)^{\text {host }}\right] } & \simeq\left[X, \Sigma_{s}^{p+q-2 n} \Sigma_{T}^{p+n}\left(\mathbf{H} \mathbb{L}^{p}\right)^{\mathbf{h o s t}}\right] \\
& \simeq\left[\Sigma_{s}^{2 n-p-q} X, \Sigma_{T}^{p+n}\left(\mathbf{H} \mathbb{L}^{p}\right)^{\mathbf{h o s t}}\right] .
\end{aligned}
$$

This is equal to

$$
L^{p+n} H^{2(p+n)-(2 n-p-q)}(X) \otimes_{\mathbb{Z}} \mathbb{L}^{p}=L^{p+n} H^{3 p+q}(X) \otimes_{\mathbb{Z}} \mathbb{L}^{p}
$$


by Theorem 8.2.5 and Section 2.3.3. This $E_{1}$-spectral sequence is actually identical to an $E_{2}$-spectral sequence after reindexing. Indeed, let

$$
\widetilde{E}_{2}^{p^{\prime}, q^{\prime}}(X, n)=L^{n-q^{\prime}} H^{p^{\prime}-q^{\prime}}(X) \otimes_{\mathbb{Z}} \mathbb{L}^{q^{\prime}} .
$$

For $r^{\prime}:=r+1$, a simple calculation shows that the equality $E_{r}^{p, q}=\widetilde{E}_{r^{\prime}}^{p^{\prime}, q^{\prime}}$ gives the equalities $p+n=n-q^{\prime}, 3 p+q=p^{\prime}-q^{\prime}, p=-q^{\prime}$ so that

$$
\begin{aligned}
E_{r}^{p+r, q-r+1} & =L^{p+r+n} H^{3 p+q+2 r+1}(X) \otimes_{\mathbb{Z}} \mathbb{L}^{-p-r} \\
& =L^{n-q^{\prime}+r} H^{p^{\prime}-q^{\prime}+2 r+1}(X) \otimes_{\mathbb{Z}} \mathbb{L}^{q^{\prime}-r}=\widetilde{E}_{r^{\prime}}^{p^{\prime}, q^{\prime}}
\end{aligned}
$$

as desired. In summary we get an analogue of Hopkins-Morel spectral sequence:

Theorem 9.0.3 For $X \in \mathbf{S m}_{\mathbb{C}}$ and $n \geq 0$, there is a spectral sequence

$$
E_{2}^{p, q}(n)=L^{n-q} H^{p-q}(X) \otimes_{\mathbb{Z}} \mathbb{L}^{q} \Rightarrow \operatorname{MGL}_{\mathrm{sst}}^{p+q, n}(X) .
$$

There is a natural morphism of spectral sequences:

$$
H_{\mathcal{M}}^{p-q}(X, \mathbb{Z}(n-q)) \otimes_{\mathbb{Z}} \mathbb{L}^{q} \Longrightarrow \operatorname{MGL}^{p+q, n}(X)
$$

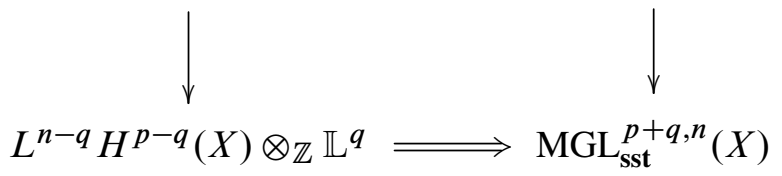

Repeating the argument for MGL smashed with mod $l$-Moore spectrum, and using that the left vertical arrow in (9.0.3) mod $l$ is an isomorphism (cf [11, Theorem 30]), we deduce that $\mathrm{MGL}^{p, q}$ and $\mathrm{MGL}_{\text {sst }}^{p, q}$ are identical with finite coefficients. On the other hand, applying Naumann, Spitzweck and Østvær [33, Corollary 10.6], we note the spectral sequence of Theorem 9.0.3 degenerates tensoring with $\mathbb{Q}$. Here is a summary:

Corollary 9.0.4 Let $X \in \mathbf{S m}_{\mathbb{C}}$ and $p, q \in \mathbb{Z}$. For $l \geq 1$, we have

$$
\operatorname{MGL}^{p, q}(X, \mathbb{Z} / l) \simeq \operatorname{MGL}_{\mathrm{sst}}^{p, q}(X, \mathbb{Z} / l) .
$$

We also have

$$
\operatorname{MGL}_{\text {sst }}^{*, *}(X) \otimes_{\mathbb{Z}} \mathbb{Q} \simeq L^{*} H_{\mathbb{Q}}^{*}(X) \otimes_{\mathbb{Z}} \mathbb{L}
$$

as graded $\mathbb{L}_{\mathbb{Q}}$-modules.

Let $\Omega_{\text {alg }}^{*}$ be the algebraic cobordism modulo algebraic equivalence given by the authors in [21]. By the universal property of $\Omega_{\text {alg }}^{*}(-)$, there is a natural functor $\Omega_{\text {alg }}^{*}(-) \rightarrow$ $\mathrm{MGL}_{\mathbf{s s t}}^{2 *, *}(-)$.

Corollary 9.0.5 The maps $\mathbb{L} \rightarrow \Omega_{\mathrm{alg}}^{*}(\mathrm{pt}) \rightarrow \mathrm{MGL}_{\mathrm{sst}}^{2 *, *}(\mathrm{pt})$ are isomorphisms. 
Proof The first map is an isomorphism by [21, Theorem 1.2(2)]. The spectral sequence in Theorem 9.0.3 shows that $\Omega_{\text {alg }}^{*}(\mathrm{pt}) \rightarrow \mathrm{MGL}_{\mathrm{sst}}^{2 *, *}(\mathrm{pt})$ is surjective. Composing with $\mathrm{MGL}_{\mathrm{sst}}^{2 * *}(\mathrm{pt}) \rightarrow \mathrm{MU}^{2 *}(\mathrm{pt})$ gives an isomorphism $\Omega_{\mathrm{alg}}^{*}(\mathrm{pt}) \simeq \mathrm{MU}^{2 *}(\mathrm{pt}) \simeq \mathbb{L}$ by [21]. In particular, the map $\Omega_{\mathrm{alg}}^{*}(\mathrm{pt}) \rightarrow \mathrm{MGL}_{\mathrm{sst}}^{2 *, *}(\mathrm{pt})$ is injective.

For the algebraic cobordism $\Omega^{*}(-)$ of Levine and Morel [25], the map $\Omega^{*}(X) \rightarrow$ $\operatorname{MGL}^{2 *, *}(X)$ is an isomorphism for $X \in \mathbf{S m}_{\mathbb{C}}$ by Levine [24]. By combining Theorem 9.0.3, Corollary 9.0.5 and the methods of [24], it is probably possible to prove that $\Omega_{\text {alg }}^{*}(X) \rightarrow \mathrm{MGL}_{\text {sst }}^{2 *, *}(X)$ is an isomorphism for $X \in \mathbf{S m}_{\mathbb{C}}$. But we do not attempt this in this paper.

Acknowledgements The authors would like to thank Aravind Asok, Denis-Charles Cisinski, Frédéric Déglise, Bertrand Guillou, Christian Haesemeyer, Marc Levine, Pablo Pelaez, Markus Spitzweck and the referee of AGT. During this work, JP was partially supported by the National Research Foundation of Korea (NRF) grant number 2012-0000796 and Korea Institute for Advanced Study (KIAS) grant, both funded by the Korean government (MEST), and TJ Park Junior Faculty Fellowship funded by POSCO TJ Park Foundation.

\section{References}

[1] S Bloch, S Lichtenbaum, A spectral sequence for motivic cohomology, preprint

[2] A K Bousfield, E M Friedlander, Homotopy theory of $\Gamma$-spaces, spectra and bisimplicial sets, from: "Geometric applications of homotopy theory, II", Lecture Notes in Math. 658, Springer, Berlin (1978) 80-130 MR513569

[3] C Chu, Morphic cohomology and singular cohomology of motives over the complex numbers, Topology Appl. 156 (2009) 2812-2831 MR2556038

[4] E M Friedlander, C Haesemeyer, M E Walker, Techniques, computations and conjectures for semitopological K-theory, Math. Ann. 330 (2004) 759-807 MR2102312

[5] E M Friedlander, H B Lawson, Jr, A theory of algebraic cocycles, Ann. of Math. 136 (1992) 361-428 MR1185123

[6] E M Friedlander, A Suslin, The spectral sequence relating algebraic K-theory to motivic cohomology, Ann. Sci. École Norm. Sup. 35 (2002) 773-875 MR1949356

[7] E M Friedlander, V Voevodsky, Bivariant cycle cohomology, from: "Cycles, transfers and motivic homology theories", Ann. of Math. Stud. 143, Princeton Univ. Press (2000) 138-187 MR1764201

[8] E M Friedlander, M E Walker, Comparing K-theories for complex varieties, Amer. J. Math. 123 (2001) 779-810 MR1854111 
[9] E M Friedlander, M E Walker, Semitopological K-theory using function complexes, Topology 41 (2002) 591-644 MR1910042

[10] E M Friedlander, M E Walker, Rational isomorphisms between $K$-theories and cohomology theories, Invent. Math. 154 (2003) 1-61 MR2004456

[11] E M Friedlander, M E Walker, Semitopological $K$-theory, from: "Handbook of $K-$ theory: Vol. 1, 2”, (E M Friedlander, D R Grayson, editors), Springer, Berlin (2005) 877-924 MR2181835

[12] PG Goerss, JF Jardine, Simplicial homotopy theory, Progress in Math. 174, Birkhäuser (1999) MR1711612

[13] J B Heller, Semitopological cobordism for complex varieties, PhD Thesis, Northwestern University (2006) Available at http://search.proquest.com/docview/ 305294461

[14] PS Hirschhorn, Model categories and their localizations, Math. Surveys and Monogr. 99, Amer. Math. Soc. (2003) MR1944041

[15] M Hovey, Spectra and symmetric spectra in general model categories, J. Pure Appl. Algebra 165 (2001) 63-127 MR1860878

[16] M Hoyois, From algebraic cobordism to motivic cohomology (2012) arXiv: 1210.7182v3 to appear in J. Reine Angew. Math.

[17] J F Jardine, Generalized étale cohomology theories, Progress in Math. 146, Birkhäuser (1997) MR1437604

[18] J F Jardine, Motivic symmetric spectra, Doc. Math. 5 (2000) 445-553 MR1787949

[19] J F Jardine, The K-theory presheaf of spectra, from: "New topological contexts for Galois theory and algebraic geometry", (A Baker, B Richter, editors), Geom. Topol. Monogr. 16 (2009) 151-178 MR2544389

[20] Y Kim, Motivic symmetric ring spectrum representing algebraic $K$-theory, $\mathrm{PhD}$ Thesis, University of Illinois at Urbana-Champaign (2010) Available at http:// search.proquest. com/docview/863644440

[21] A Krishna, J Park, Algebraic cobordism theory attached to algebraic equivalence, J. K-Theory 11 (2013) 73-112 MR3034284

[22] T Lawson, Motivic homotopy: Notes from the lectures by M Hopkins, week 8 Available at http://www.math.umn.edu/ tlawson/papers/motivic.html

[23] M Levine, The homotopy coniveau tower, J. Topol. 1 (2008) 217-267 MR2365658

[24] M Levine, Comparison of cobordism theories, J. Algebra 322 (2009) 3291-3317 MR2567421

[25] M Levine, F Morel, Algebraic cobordism, Springer, Berlin (2007) MR2286826

[26] S Mac Lane, Categories for the working mathematician, 2nd edition, Graduate Texts in Math. 5, Springer, Berlin (1998) MR1712872 
[27] C Mazza, V Voevodsky, C Weibel, Lecture notes on motivic cohomology, Clay Math. Monogr. 2, Amer. Math. Soc. (2006) MR2242284

[28] J McCleary, A user's guide to spectral sequences, 2nd edition, Cambridge Studies in Advanced Math. 58, Cambridge Univ. Press (2001) MR1793722

[29] S A Mitchell, Hypercohomology spectra and Thomason's descent theorem, from: "Algebraic K-theory", (V P Snaith, editor), Fields Inst. Commun. 16, Amer. Math. Soc. (1997) 221-277 MR1466977

[30] F Morel, The stable $\mathbb{A}^{1}$-connectivity theorems, $K$-Theory 35 (2005) 1-68 MR2240215

[31] F Morel, $\mathbb{A}^{1}$-algebraic topology over a field, Lecture Notes in Math. 2052, Springer (2012) MR2934577

[32] F Morel, V Voevodsky, $\mathbb{A}^{1}$-homotopy theory of schemes, Inst. Hautes Études Sci. Publ. Math. (1999) 45-143 MR1813224

[33] N Naumann, M Spitzweck, P A Østvær, Motivic Landweber exactness, Doc. Math. 14 (2009) 551-593 MR2565902

[34] P A Østvær, O Röndigs, V Voevodsky, Voevodsky's Nordfjordeid lectures: Motivic homotopy theory, from: "Motivic homotopy theory", Springer (2007) 147-221 MR2334215

[35] I Panin, Oriented cohomology theories of algebraic varieties, K-Theory 30 (2003) 265-314 MR2064242

[36] O Röndigs, P A Østvær, Modules over motivic cohomology, Adv. Math. 219 (2008) 689-727 MR2435654

[37] M Spitzweck, Relations between slices and quotients of the algebraic cobordism spectrum, Homology, Homotopy Appl. 12 (2010) 335-351 MR2771593

[38] M Spitzweck, Slices of motivic Landweber spectra, J. K-Theory 9 (2012) 103-117 MR2887201

[39] A Suslin, V Voevodsky, Relative cycles and Chow sheaves, from: "Cycles, transfers and motivic homology theories", Ann. of Math. Stud. 143, Princeton Univ. Press (2000) 10-86 MR1764199

[40] R W Thomason, T Trobaugh, Higher algebraic $K$-theory of schemes and of derived categories, from: “The Grothendieck Festschrift, Vol. III”, (P Cartier, L Illusie, N M Katz, G Laumon, K A Ribet, editors), Progr. Math. 88, Birkhäuser, Boston (1990) 247-435 MR1106918

[41] V Voevodsky, $\mathbb{A}^{1}$-homotopy theory, from: "Proc. of the International Congress of Math., Vol. I", Doc. Math. (1998) 579-604 MR1648048

[42] V Voevodsky, On the zero slice of the sphere spectrum, Tr. Mat. Inst. Steklova 246 (2004) 106-115 MR2101286 
School of Mathematics, Tata Institute of Fundamental Research

1 Homi Bhabha Road, Colaba, Mumbai 400 005, India

Department of Mathematical Sciences, KAIST

291 Daehak-ro, Yuseong-gu, Daejeon, 305-701, South Korea

amal@math.tifr.res.in, jinhyun@mathsci.kaist.ac.kr, jinhyun@kaist.edu

Received: 20 January $2014 \quad$ Revised: 26 August 2014 
